\title{
Management of children and young people with idiopathic thickened pituitary stalk and/or idiopathic central diabetes insipidus: A national clinical practice consensus guideline
}

Manuela Cerbone ${ }^{1,2}$, MD · Johannes. Visser ${ }^{3}$, FRCPCH $\cdot$ Chloe Bulwer ${ }^{4}$, MBBS $\cdot$ Ashraf Ederies ${ }^{5}$, MD $\cdot$ Kirtana Vallabhaneni ${ }^{6}$, MBBS · Prof Stephen Ball ${ }^{7}$, FRCP · Prof Ian Kamaly-Asl ${ }^{8}$, FRCS · Prof Ashley Grossman $^{9,10}$, FMedSci · Helena Gleeson ${ }^{11}$, MD · Prof Márta Korbonits ${ }^{12}$, FRCP · Vasanta Nanduri ${ }^{13}$, FRCPCH · Vaya Tziaferi ${ }^{14}$, MD · Prof Tom Jacques ${ }^{15}$, FRCPath · Helen A. Spoudeas ${ }^{1,2}$, FRCPCH

${ }^{1}$ London Centre for Paediatric Endocrinology and Diabetes at Great Ormond Street Children's Hospital and University College London Hospitals

${ }^{2}$ Section of Molecular Basis of Rare Disease, Genetics and Genomic Medicine Programme, University College London Great Ormond Street Hospital Institute of Child Health

${ }^{3}$ Cambridge University Hospitals NHS Foundation Trust

${ }^{4}$ Child Health, Royal Devon and Exeter Hospital

${ }^{5}$ Doha (previously Great Ormond Street Hospital)

${ }^{6}$ Whittington Health Trust (previously UCL Medical School)

${ }^{7}$ University of Manchester \& Manchester University Foundation Trust

${ }^{8}$ Royal Manchester Children's Hospital and The University of Manchester

${ }^{9}$ Oxford Centre for Diabetes, Endocrinology and Metabolism, University of Oxford

${ }^{10}$ Centre for Endocrinology, Barts and the London School of Medicine, Queen Mary University of London

${ }^{11}$ Queen Elizabeth Hospital, Birmingham

${ }^{12}$ Barts and the London School of Medicine, Queen Mary University of London

${ }^{13}$ Watford General Hospital

${ }^{14}$ AHEPA University Hospital, Thessaloniki, Greece (previously University Hospitals of Leicester)

${ }^{15}$ Great Ormond Street Hospital NHS Foundation Trust

\section{Corresponding author}

Dr Manuela Cerbone, MD

Clinical Research Fellow

University College London Great Ormond Street Hospital Institute of Child Health

30 Guilford St, Holborn, London WC1N 1EH, UK

Email: manuela.cerbone@ucl.ac.uk

Tel: +442079052283

Key words: Pituitary - stalk - thickening - tumour - diabetes insipidus- guidelines

Short title: Management of idiopathic pituitary stalk thickening and central diabetes insipidus 


\section{Summary}

Unexplained/idiopathic pituitary stalk thickening (iTPS) or central diabetes insipidus (iCDI) can not only harbour rare occult malignancies in $40 \%$ of children and young people under 19 years (CYP), but also reflect benign congenital defects. Between 2014 and 2019, a multidisciplinary expert national Guideline Development Group (GDG) systematically developed a management flow chart and clinical practice guideline to inform specialist care and improve outcomes.

All cases of iTPS and/or iCDI require optometry, dynamic pituitary function testing, specialist pituitary imaging, serum $\beta$-human choriogonadotropin $(\beta-\mathrm{HCG})$ and $\alpha$-foetoprotein $(\alpha \mathrm{FP})$ levels, chest $\mathrm{x}$-ray, abdominal ultrasound and skeletal survey for occult disease. Stalk thickening $4 \mathrm{~mm}$ or more at the optic chiasm and/or 3 $\mathrm{mm}$ or more at pituitary insertion is potentially pathological, particularly if an endocrinopathy or visual impairment co-exist. The role of surveillance, cerebrospinal fluid tumour markers and whole-body imaging, indications, timing and risks of stalk biopsy, and criteria for discharge, are defined. A registry of outcomes to validate the systematic approach described in this guideline and research to determine paediatric normative stalk sizes and the possible role of novel biomarkers and/or imaging techniques in diagnosis are encouraged. 


\section{Key messages}

1. The challenge posed by apparently iTPS or iCDI in CYP is to differentiate congenital variants from those harbouring occult neoplasia. Unlike in adults, hypophysitis and neurosarcoidoisis are exceptionally rare, whilst genetic CDI and congenital midline brain defects are a real possibility.

2. The 39 recommendations and decision-making flow chart aim to determine which cases are likely to herald occult oncological disease [usually where TPS/CDI and endocrinopathy or visual impairment co-exist] and which may be discharged after stable pituitary MRI and clinical surveillance [usually mild/stable TPS alone].

3. To consider a pituitary stalk as thickened, the GDG defined cut-offs of $4 \mathrm{~mm}$ or more at the optic chiasm or $3 \mathrm{~mm}$ or more at pituitary insertion. However, without paediatric norms, size alone cannot distinguish physiological from pathological variants. If there is co-existing pituitary or visual dysfunction, even lower cut-off criteria ( $3 \mathrm{~mm}$ or $2 \mathrm{~mm}$, respectively) may warrant further assessment.

4. Minimally invasive $1^{\text {st }}$ line investigations are strongly recommended in all such cases:

i) serum $\beta$-hCG and $\alpha \mathrm{FP}$ [to detect secreting germ cell tumour (GCT)]

ii) chest X-ray, abdominal ultrasound and skeletal survey (to detect signs of Langerhans cell histiocytosis ( $\mathrm{LCH})$ and possible sites for diagnostic biopsy)

iii) dynamic anterior and posterior pituitary function [to detect occult growth hormone (GH) and adrenocorticotrophin (ACTH) deficiency or CDI]

iv) optometry (visual acuity and field assessment) especially if TPS encroaches on the optic chiasm

5. CSF tumour markers ( $\beta-h C G$ and $\alpha \mathrm{FP})$ and whole-body imaging are $2^{\text {nd }}$ line investigations recommended in cases where stalks are large $(>6 \cdot 5-7 \cdot 0 \mathrm{~mm}) /$ enlarging and/or there is evolving pituitary or visual dysfunction.

6. Pituitary stalk biopsy should only be undertaken in specialist multidisciplinary centres, in selected cases with endocrine or visual symptoms whose $2^{\text {nd }}$ line investigations have been negative, and whose stalk thickening is sufficient $(>6 \cdot 5-7 \cdot 0 \mathrm{~mm})$ to yield a diagnostic biopsy without further visual or endocrine harm.

7. More research is needed to further define normative criteria for pituitary stalks in CYP and the role of novel imaging techniques and biomarkers in securing a diagnosis without resorting to a pituitary biopsy. 


\section{Introduction}

Pituitary stalk thickening (TPS) and central diabetes insipidus (CDI) are rare conditions (2 to 4 per 100,000 for CDI) (1) which occur independently or synchronously/metachronously. Children and Young People before their $19^{\text {th }}$ birthday (CYP) with TPS or CDI of indeterminate aetiology (iTPS or iCDI), represent a diagnostic conundrum to differentiate the occult harmful, yet treatable, oncological, inflammatory and infectious aetiologies, from benign congenital conditions.

Diagnostic criteria for defining TPS are controversial, complicated by incidental findings, imprecise stalk measurement at differing levels of the stalk and lack of age-appropriate norms. There is limited published clinical experience, especially in CYP. Our systematic review identified significantly different prevalences of underlying aetiologies in CYP (Table 1), compared to adults (2), and a lack of high quality or randomised trials in both. This, their rarity and presentation to diverse specialties, causes unacceptable inequalities in care. Under the auspices of the paediatric oncology (Children's Cancer and Leukaemia Group - CCLG) and paediatric endocrine (British Society for Paediatric Endocrinology and Diabetes - BSPED) societies, our objective was to develop a nationally endorsed clinical practice guideline for the investigation, management and follow-up of CYP with iTPS and/or iCDI, to standardise service provision and improve outcomes. 


\section{Methods}

A national GDG comprised of clinical experts across the UK in adult and paediatric endocrinology, oncology, neuroradiology, neuropathology, and neurosurgery was convened in 2014.

The guideline was developed using AGREE II methodology (3).

The guideline objectives were summarised in 64 PICO (Population, Intervention, Comparison, Outcome)

clinical questions (4), reviewed by UK stakeholders, prior to a systematic literature search (Fig. 1).

We systematically searched Ovid MEDLINE, PubMed, EMBASE, and Cochrane Library databases on English language papers meeting our inclusion criteria published between January 1990 and October 2014 and updated this from November 2014 - July 2019 (Fig. 1).

Selected papers were appraised using GRADE criteria (5). High quality evidence was lacking. Where there was little or no paediatric evidence, the GDG considered adult studies, and downgraded the evidence level accordingly.

First, the GDG reviewed the likelihood of different occult aetiologies underlying iTPS or iCDI in CYP, finding it differed significantly from adults (2). The evidence - after an average of 6 (2-10) years surveillance across 11 studies - is summarised for the first time in Table 1. Neoplasia (45.5\%) - either Langerhans Cell Histiocytosis (LCH) $(16 \cdot 1 \%)$, Germ Cell Tumours (GCT) $(13 \cdot 1 \%)$ or craniopharyngiomas $(12 \cdot 3 \%)$ - accounted for the majority, congenital lesions was the next largest group $(19 \cdot 1 \%)$, whilst one third $(29 \cdot 1 \%)$ remained idiopathic. Only a minority had infectious or inflammatory/autoimmune aetiologies, more common in adults.

Thirty-nine recommendations were made.

Where a moderate evidence base existed, the GDG made 9 guideline recommendations. Where an evidence base was lacking, or to add support for moderate evidence, the GDG put forward recommendations to an independent international panel of up to 22 (minimum 11) experts consulted in 2 rounds of a Delphi consensus process (6). A recommendation was accepted if supported by over $70 \%$ of Delphi respondents with relevant specialty expertise to provide an opinion: 23 recommendations achieved $>75 \%$ agreement in the $1^{\text {st }}$ round; 9 of these were reformulated or separated based on the panel's comments and put to a $2^{\text {nd }}$ round. This resulted in 29 consensus recommendations, and one further recommendation was made by GDG consensus alone. Recommendations were all based on a trade-off between benefits and harms, the quality of evidence, stakeholder and user feedback, and two independent expert peer reviews.

We followed a consistent NICE terminology, using the verbs "offer" and "consider" respectively, for strong and less strong interventions/actions, and the verbs "should" for strong, "may" and "consider" for moderate, and "note" for weak recommendations. The evidence levels are shown as low (I) or medium (II) quality or a combination (I/II), in addition to any Delphi or GDG consensus. Six strong consensus recommendations were felt clinically implicit or ethically necessary to avoid patient harm and no evidence levels are reported for these. Research Recommendations are areas prioritised by the GDG for further research. The guideline was ultimately endorsed by the Quality Improvement Committee of the RCPCH and an update with literature review is planned in 5 years. 


\section{Recommendations and explanations}

\section{Panel 1: Service Provision}

- Offer age-appropriate care, provided by an endocrinologist in a specialist centre with expertise in managing pituitary tumours, to all CYP with iTPS and/or iCDI. (I/II, Delphi 100\%)

- The endocrinologist providing care for those with iTPS and/or iCDI should liaise closely with the specialist Multidisciplinary Team (MDT) for pituitary tumours with mandated specialists from paediatric and adult endocrinology, pituitary surgery, neuroradiology, neuropathology and neurooncology. (I/II, Delphi 100\%)

- Given the rarity of pituitary tumours in CYP, a national clinical database and facilitated centralised review of images, histology and decision-making process should be developed. (Delphi 90\%)

- A centralised, national, pituitary MDT may require commissioning to facilitate review of complex cases. (Delphi 90\%)

- Offer all patients the opportunity to contribute to tissue banking and relevant ethically approved national and international biology and treatment studies. (Delphi 100\%)

Evolving pituitary dysfunction over a variable timeframe is well reported in CYP with iTPS with/without iCDI (7-10). Hence, the GDG favoured early referral to a specialist pituitary centre with an age-appropriate lead endocrinologist coordinating care, ensuring anterior and posterior pituitary assessment, and timely diagnosis and treatment of occult life-threatening endocrinopathies which affect decision making. These, and baseline optometric and radiological assessments, should be discussed at the pituitary and neuro-oncology MDT to agree management.

The limited evidence base and rarity of the condition with differing aetiology in CYP from adults, persuaded the GDG, Delphi panel and peer experts, that both a centralised pituitary MDT and a national registry would inform and help standardise care. A national forum which could form the basis of a national pituitary MDT has been operational for complex cases since 2010 (11). Such an MDT could also facilitate participation in national/international biology (with tissue banking) and treatment studies.

\section{Panel 2: Initial imaging and clinical assessment}

\section{Defining criteria for pituitary stalk thickening}

- $\quad$ Consider that a pituitary stalk (assessed by dedicated pituitary imaging) may be pathologically thickened and require further investigation and MRI surveillance, if there is uniform or focal thickening, in the sagittal and/or coronal plane, measuring $3 \mathrm{~mm}$ or more at pituitary insertion and/or $4 m m$ or more at the optic chiasm. (I/II, Delphi 100\%)

- Consider further investigation and MRI surveillance for stalks measuring between 2 and $3 \mathrm{~mm}$ at the pituitary insertion and/or between 3 and $4 \mathrm{~mm}$ at the level of optic chiasm if there are associated clinical features that increase the risk of pathology (such as CDI, anterior pituitary dysfunction or visual deficits). (I/II)

- $\quad$ The interpretation of the stalk appearances requires neuroradiological expertise. Given the absence of age-specific norms and the inter-and intraindividual variability, size criteria alone do not always differentiate between pathological and physiological variants. (I/II)

\section{Dedicated pituitary MRI to detect pituitary stalk thickening}

- $\quad$ Offer head and dedicated pituitary MRI to all CYP with suspected TPS and/or CDI. This should include uncontrasted $2 D$ thinly sliced $(<3 \mathrm{~mm})$ with no gap T1 and T2 weighted images in sagittal and coronal planes (and ideally at least one 3D highly weighted T2 sequence) to assess the possibility of uniform and/or focal thickening of the pituitary stalk in both planes. (II, Delphi 100\%)

Additional MRI findings which increase suspicion of pathology 
- The additional absence of a pituitary bright spot on the T1 non-contrasted scan and/or a significant reduction or enlargement of the pituitary, though not diagnostic, should increase suspicion of pathology. (II, Delphi 100\%)

- Consider a disease-tailored diagnostic approach if extra-pituitary MRI findings suggest a specific underlying aetiology (e.g. skull lesions in LCH and pineal lesions in bifocal germinoma or LCH). (II) Systematic history and clinical evaluation

- If the aetiology for a confirmed TPS and/or CDI is not apparent at presentation and a systematic history and clinical evaluation assessing the commonest causes have failed to reveal a potential focus for testing, or if focussed testing has proved uninformative, a stepwise decision-making approach for investigation and surveillance should be adopted in all patients (Fig. 2). (GDG consensus)

The stalk tapers from its top level at the optic chiasm to its lower pituitary insertion; so, measurements at upper, middle and lower levels are required to capture its normal shape and size (Fig. 3) and the degree and shape of any abnormal thickening (Fig. 4). As there are no differences between the antero-posterior and transverse diameters at all levels of measurement (12-16), the thickening of the pituitary stalk can be assessed in any direction as long as the measurements are performed in the same plane and at the same level within a protocol applied in each single centre for stalk abnormalities.

In the absence of such normative data in children, the GDG derived the dimensions of a "normal" pituitary stalk from dedicated pituitary imaging in healthy late adolescents and adult volunteers using computed tomography (12), 1.5 Tesla (T) MRI $(13,14)$ and 3T MRI $(15)$; the upper normative limits ranged between $2 \mathrm{~mm}$ and $4 \cdot 5$ $\mathrm{mm}$. Satogami et al. (15) measured the stalk at both pituitary insertion and optic chiasm on T2-weighted oblique-axial fast spin-echo images in volunteers aged 21 to 43 years. This study-reported normal dimensions of $2.32 \pm 0.39 \mathrm{~mm}$ and $2 \cdot 16 \pm 0.37 \mathrm{~mm}$ for anterior-posterior and transverse diameters respectively, at pituitary insertion, and $3.25 \pm 0.43 \mathrm{~mm}$ and $3.35 \pm 0.44 \mathrm{~mm}$ respectively, at the optic chiasm. However, Godano et al. (16), in the only paediatric study of 102 children aged 7-3-12 years, documented smaller stalk norms - between 2.35 and $2.82 \mathrm{~mm}$ proximally, 1.79 and $2.45 \mathrm{~mm}$ at midpoint and 1.28 and $1.78 \mathrm{~mm}$ distally - on T1 precontrast, T1 post-contrast, and T2-DRIVE images. However, the GDG considered these data flawed for the purpose of providing normative stalk size data in children, since the 'healthy' group included patients with congenital hypopituitarism, who, though not displaying major hypothalamo-pituitary abnormalities (without TPS and with eutopic posterior pituitary), could still have pathological congenital hypoplastic or threadlike stalks. Hence this group cannot be considered representative of the healthy population and the measurements provided might err on the lower side.

Thus, to increase the positive predictive value, the consensus was to define a TPS in CYP as measuring $3 \mathrm{~mm}$ or more at pituitary insertion and/or $4 \mathrm{~mm}$ or more at the optic chiasm, like most prior paediatric pathological case series. However, in the presence of clinical signs such as pituitary and/or visual disturbance, even smaller stalk sizes - between $2 \mathrm{~mm}$ and $3 \mathrm{~mm}$ at pituitary insertion and/or between $3 \mathrm{~mm}$ and $4 \mathrm{~mm}$ at the optic chiasm - may have pathological significance.

To differentiate pathological thickening from physiological variants, interpretation requires dedicated imaging, neuroradiological expertise and multidisciplinary assessment as well as size criteria. While very large stalks $(6 \cdot 5-7 \cdot 0 \mathrm{~mm}$ or more) are known to be more predictive of neoplasms $(7-10,17)$, the predictive value of lesser degrees of thickening is less clear, whilst how this evolves over time and its correlation with pituitary or visual deficits is unknown.

Both head and dedicated pituitary MRI are essential to accurately characterise the stalk size and shape (16), avoid artefactual misinterpretation and detect any congenital malformations.

Godano et al (16) favour high-resolution heavily T2-weighted sequences (such as sagittal T2 DRIVE which takes $<3$ minutes to acquire, or CISS or FIESTA) to precisely measure the stalk in the sagittal plane and identify its abnormalities, without the addition of gadolinium contrast (16). This, and the potential role for the so called "mismatch pattern" (consisting of discrepancies between T2-DRIVE and postcontrast T1-weighted images of PS thickness) as a prognostic marker of likelihood of evolving future anterior pituitary dysfunction or pituitary stalk stability in patients with CDI (18), is promising, but requires confirmation in further studies. 
Standard MR brain sequences must accompany the dedicated pituitary sequences to provide supporting information towards a diagnosis. Given the potential to deposit in the eloquent brain structures and in other solid organs, the use of gadolinium-based contrast medium in not routinely recommended in all children presenting with idiopathic TPS/CDI. However, its use is specifically indicated to rule out secondary brain metastases in cases where the clinical course is increasingly suspicious of a high-grade tumour, such as a germinoma (i.e. additional precocious pseudo-puberty, raised tumour markers, raised intracranial pressure, concomitant localisation in the pineal gland, basal ganglia calcification, visual abnormalities).

Additional pituitary and extra-pituitary abnormalities may aid diagnosis and should be noted (e.g. skull lesions suggestive of LCH or pineal lesions suggestive of bifocal germinoma or LCH). An absent posterior pituitary bright spot and/or anterior pituitary hypoplasia increase suspicion of pathology (19), although the former finding has also been observed in nephrogenic DI (20) and in 4.1\% of patients undergoing a brain MRI for nonendocrinological reasons (21). Suprasellar midline anomalies may indicate developmental or syndromic conditions (22). Whilst its specific prevalence in CDI is unknown, pituitary hypoplasia has been described in $98 \%$ of patients with isolated hypopituitarism and $83.3 \%$ of those with associated midline brain defects and/or optic nerve hypoplasia (22). It has also been described in $24 \%$ of LCH cases (23), whilst hypophysitis (24) and germinomas $(25,26)$ can present with pituitary enlargement.

Systematic history and clinical examination, focussed on the common differential diagnoses in CYP (Table 1 and 2), should be undertaken at diagnosis and repeatedly during follow up. If the diagnosis remains occult, we recommend a stepwise decision-making and surveillance approach (Fig. 2).

\section{Panel 3: Subsequent stepwise approach}

\section{A) First line investigations}

Serum tumour markers, haematology, liver and renal function

- Offer measurement of serum $\beta-h C G$ and $\alpha F P$ to all CYP with radiologically confirmed iTPS and/or iCDI. (II, Delphi 92\%)

- Although non-specific, consider performing Erythrocyte Sedimentation Rate (ESR), Full Blood Count $(F B C)$, liver function, urea, creatinine, and electrolytes, to aid the diagnostic process. (Delphi 92\%)

Endocrinology

- Offer an early endocrine assessment of growth and pubertal status, posterior pituitary function and baseline and dynamic tests of anterior pituitary function, including GH and ACTH reserve, to all CYP with iTPS and/or iCDI. (II, Delphi 100\%)

Ophthalmology

- Offer a formal baseline assessment of visual acuity and, if child is able to co-operate, visual fields by optometry, to all CYP with iTPS and/or iCDI, especially if the TPS is proximal to, or abutting the chiasm. (II, Delphi 100\%)

\section{Imaging}

- Offer a skeletal survey, abdominal ultrasound and a chest $x$-ray (CXR) to all CYP with iTPS and/or iCDI in whom initial blood tests have failed to reveal the aetiology. (II, Delphi 87\%)

Investigations of specific conditions as clinically indicated

Infectious and inflammatory/autoimmune disease

- Consider testing for Tuberculosis (TB) or autoimmune disease, as per local practice, if indicated by history and clinical examination. (I/II, Delphi 92\%)

- Serum Angiotensin-Converting Enzyme (ACE) to screen for neurosarcoidosis in CYP with iTPS and/or iCDI is not recommended. (I/II, Delphi 100\%)

- Congenital midline brain abnormalities [also called Septo-Optic Dysplasia (SOD) spectrum]

- Offer genetic counselling and, where appropriate, molecular genetic testing for SOD spectrum, if imaging (midline anomalies, optic nerve hypoplasia), age and ophthalmology are consistent with this diagnosis. (II)

Familial CDI 
- Offer genetic counselling and genetic testing for inherited forms of CDI in CYP with isolated CDI and neither TPS nor other midline neuroimaging abnormalities suggestive of SOD, especially if there is a family history and/or early childhood presentation. (II, Delphi 100\%)

\section{B) Second line investigations}

\section{Lumbar puncture}

Indications

- Consider a diagnostic lumbar puncture if, after initial blood tests and imaging, the aetiology is not apparent and the patient meets one or more of the following criteria a) iTPS $\geq 6 \cdot 5-7 \cdot 0 \mathrm{~mm}$ or progressively enlarging over time b) iTPS associated with iCDI c) iTPS associated with evolving anterior pituitary deficiencies and/or pituitary enlargement and/or deteriorating visual function. (II, Delphi 93\%)

Markers of GCT and LCH

- When a diagnostic lumbar puncture is undertaken, offer measurement of $\beta$ - $h C G$ and $\alpha F P$ in the CSF, together with CSF cytology. (II, Delphi 100\%)

Tuberculosis and neurosarcoidosis

- Consider CSF analysis for TB only in those at risk. (II, Delphi 78\%)

- Consider CSF ACE only if neurosarcoidosis is strongly suspected. (I, Delphi 78\%)

\section{Imaging}

- Consider whole body imaging to detect distant, occult LCH lesions more amenable to biopsy, in CYP whose TPS and/or CDI remain idiopathic after initial blood tests, imaging and CSF screening, but are nevertheless concerning for neoplasia (i.e. TPS is $\geq 6 \cdot 5-7 \cdot 0 \mathrm{~mm}$, and/or is progressive, and/or is associated with CDI, changes in pituitary size, evolving endocrinopathy, or deteriorating visual function). (II, Delphi 90\%)

- Whole body imaging may consist of FluoroDeoxy Glucose Positron Emission Tomography MRI/CT $\left({ }^{18}\right.$ FDG PET MRI or ${ }^{18}$ FDG PET CT) or whole-body MRI, depending on local availability. (II, Delphi 90\%)

\section{C) Biopsy}

- In CYP who continue to pose a diagnostic dilemma after appropriate serial neuroimaging, whole body imaging and, if necessary, repeat CSF testing, consider a biopsy of the TPS if there is a very large $(\geq 6 \cdot 5-7 \cdot 0 \mathrm{~mm})$ or progressively enlarging stalk and/or evolving hypopituitarism and/or visual deterioration. (I/II, Delphi 100\%)

- A biopsy should only be undertaken if the TPS is judged by the MDT to be of sufficient size to yield a diagnostic sample and the benefits outweigh the risk of the procedure. (Delphi 100\%)

- Pituitary surgery on CYP should be undertaken by a pituitary surgeon nominated by the MDT. There should be ready access to transsphenoidal, endoscopic and base of skull techniques and readilyavailable age-appropriate endocrine support. (I, Delphi 90\%)

Although the sensitivity and specificity of plasma $\beta-\mathrm{hCG}$ and $\alpha \mathrm{FP}$ in detecting secreting germinomas is unknown, these tumour markers are standard assessments in such patients. Their detectability and predictive value increase with repeated sampling over time, often coupled with increased stalk thickening $(7,8,27)$. Cutoff values for marker positivity differ between studies $(28,29)$, highly sensitive $\beta$-hCG assays increasing the sensitivity of disease detection $(30,31)$. Baseline ESR, FBC, urea, creatinine, electrolytes and liver function tests are included as surrogate markers of disease severity (eg LCH or inflammatory disease), and of fluid and electrolyte homeostasis in occult CDI.

Robust evidence supports frequent anterior pituitary deficits in children with iTPS or iCDI $(9,17)$ and timely comprehensive endocrine assessment will aid diagnosis and replacement therapy of occult GH deficiency, potentially life-threatening ACTH insufficiency and CDI, and normalise growth and pubertal development. Patients with isolated TPS may have occult CDI, especially if there is additional cortisol or thyroid deficiency which their replacement may unmask. The constellation and evolving hierarchy of endocrine deficits may assist differentiation of "organic" and "idiopathic" TPS and of "genetic" and "non-genetic" CDI (Fig. 2). Anterior pituitary deficits are more likely in cases with larger stalks (32) and in organic TPS (vs idiopathic forms) $(9,10$, 
32), whilst genetic CDI is usually not associated with anterior pituitary deficits (17). It is well-known that secreting germ-cell tumours can present with precocious pseudopuberty.

Pathologies underlying TPS or CDI may extend to the optic pathways and cause visual dysfunction. In a retrospective review of 53 children, $10 \%$ with isolated iTPS, $22 \%$ with isolated iCDI and $8 \%$ with both iTPS and iCDI, presented with visual symptoms (10). This increased to $35 \%$ over time only in those with both iTPS and iCDI (10), suggesting that all patients, especially the latter group, need close ophthalmology monitoring. The rate of detection of extra-pituitary LCH, using different imaging modalities in CYP with iTPS or iCDI, is unknown. However, in CYP with known $\mathrm{LCH}, 76 \cdot 8 \%$ and $7 \cdot 7 \%$ have skeletal and hepatic disease respectively (33). A possible bony LCH lesion is more accessible to diagnostic biopsy than the pituitary stalk. Hepatomegaly or splenomegaly is suspicious of LCH or other multisystem disease, whilst a CXR may identify pulmonary involvement seen in $7 \cdot 6 \%$ of LCH cases $(33,34)$ and in $50 \%$ of tuberculous meningitis cases, a rare cause of iTPS or iCDI (35).

Unlike in adults, infectious and inflammatory or autoimmune diseases are rare causes of iTPS or ICDI in CYP (Table 1). Thus, screening tests for their detection are likely to be of very low yield unless there are strong clinical indicators. Tuberculin skin testing and plasma Interferon-Gamma Release Assays may provide evidence of prior TB but are insensitive and non-specific in diagnosing active tuberculous central nervous system (CNS) disease, which requires cerebrospinal fluid (CSF) mycobacterial studies (36).

In adults with autoimmune hypophysitis, $96 \%$ have TPS and $72 \%$ have CDI $(37,38)$, but hypophysitis itself is rare in CYP (39) (Table 1). A definitive diagnosis relies on histology since neuroimaging findings are nondiscriminatory and anti-pituitary and anti-hypothalamic antibodies $(40,41)$ lack sensitivity and specificity (39). IgG4-related hypophysitis - a new entity of pituitary dysfunction and TPS, usually with multiorgan involvement (42). As yet only one of 76 described cases affected the CYP population (one 16 year old) (43), hence routine measurement of IgG4 levels are not currently recommended.

Although frequently reported in adults with iTPS (2), sarcoidosis is exceptionally rare in CYP and serum ACE lacks sensitivity and specificity to detect neurosarcoidosis $(44,45)$. Hence, we do not recommend its routine use in CYP.

Midline neuroimaging abnormalities and/or optic nerve hypoplasia associated with CDI in a young child should raise suspicion of SOD spectrum $(10,46)$. Causative mutations are identified in under $20 \%$ of such cases $(47$, 48), but SOD may account for between $3 \cdot 4 \%$ and $14 \cdot 3 \%$ of patients with CDI (Table 1). Thus, genetic counselling with appropriate testing should be offered in these cases.

Familial CDI accounts for 2\% (10) to $7 \cdot 5 \%$ (17) of CDI in CYP (Table 1). Isolated and early-presenting cases, should prompt a careful family history, genetic counselling and a targeted genetic analysis for a dominant or, rarely, a recessive variant in the arginine vasopressin $(A V P)$ gene, particularly if symptoms are familial. $(49,50)$. Wolfram syndrome due to biallelic mutations in WSF1 gene can also cause familial CDI (50). PCSK1 mutations resulting in severe malabsorptive diarrhoea, growth hormone deficiency, central hypothyroidism, central hypogonadism and central hypocortisolism have also been recently associated with clinical CDI in $80 \%$ of these cases (50). In the rare X-linked form currently no known genes have been identified.

CYP with iTPS and/or iCDI whose aetiology remains occult after the above tests, and who in addition manifest significant or progressive TPS, endocrinopathies or visual compromise, may harbour neoplasia. In two case series comprising 53 children followed for some four years, only those with both iTPS and iCDI harboured neoplasia which was detected later $(8,10)$. In cases of isolated iCDI, the risk is less clear, not least because iTPS may additionally evolve. In one series, $40 \%$ of 38 cases with isolated iCDI developed neoplasia 1.42 to 21.83 years later, but $45 \%$ of these also developed iTPS during surveillance (10). Another series reported neoplasia in $0 / 12$ cases with isolated iCDI as compared with $4 / 10$ cases with both iCDI and iTPS after $0 \cdot 6$ to $15 \cdot 3$ years (17). Thus, the GDG considered iCDI with iTPS more indicative of underlying pathology requiring a lumbar puncture than iTPS alone, whilst the evidence for isolated iCDI is inconsistent. Nevertheless, since both series report evolving anterior pituitary dysfunction more commonly in those harbouring neoplastic disease, compared with those who don't ( $85 \%$ vs $39 \%$ (4) and $67 \%$ vs $39 \%$ (10)), and visual deterioration also tracks with neoplasia (10), the presence of these signs with iTPS decreases the threshold for CSF sampling.

Using CSF levels of $\beta$-hCG, $\alpha \mathrm{FP}$ and cytology to detect GCT is well established, but their sensitivity and specificity in iTPS or iCDI is unknown. Mildly elevated CSF $\beta$-hCG is detected in up to $38 \%$ of suprasellar 
germinomas while the majority of these patients have normal serum $\beta H C G$ levels (51). Significantly raised $\beta$ $\mathrm{hCG}$ or raised $\alpha \mathrm{FP}$ (in the context of TPS) indicates the presence of choriocarcinoma and yolk sac tumour respectively, or the presence of a mixed malignant germ cell tumour. The tumour marker levels at which the diagnosis of these conditions can be made is still debated (29). It is of note that mildly elevated CSF $\beta$-hCG levels have also been described in LCH and craniopharyngioma $(52,53)$.

Several authors have recently explored novel disease markers in patients with brain tumours. Okamoto et al (54) suggest Fluid-Placental Alkaline Phosphatase (FLAP) can differentiate intracranial germ cell tumours from other types of brain tumours and detect disease recurrence, whilst Murray et al (55) highlight how microRNA quantification may assist the non-invasive diagnosis, prognostication and management of such patients. BRAFV600E alterations have been identified in various types of primary brain tumours (56) and their detection in the serum, plasma and CSF of children with brain tumours is under investigation (57); for example, whether its detection in the CSF of patients with LCH might indicate increased risk for LCH-associated neurodegeneration (58). These and other novel markers are recommended for further research.

Given the rarity of CNS tuberculous disease in causing iTPS and/or iCDI in CYP, routine CSF examination for acid-fast bacilli (36) is not recommended, but should be considered in high-risk or exposed cases. Because the diagnostic yield is lower in children than in adults (15-20\% vs $80 \%)$ and critically dependent on CSF volume, repeated CSF examinations and commercial nucleic acid amplification assays may be needed to confirm the diagnosis where there is a high index of suspicion, whilst a tissue biopsy from a tuberculoma has a higher diagnostic yield (36).

Neurosarcoidosis is unlikely to be the cause of iTPS or iCDI in CYP, and given the inconsistent reports on the clinical utility of ACE measurement in the CSF $(44,59)$, this is not recommended unless neurosarcoidosis is strongly suspected.

Whole body (WB) imaging with ${ }^{18} \mathrm{FDG}$ PET MRI/CT or MRI are not routinely used to stage patients with LCH but may detect extracranial lesions not otherwise identified (34, 60-62). If abnormalities outside the TPS are detected and suspicious, these may be easier targets for diagnostic biopsy. The accuracy of these imaging modalities to identify such lesions in this context is uncertain and which whole body imaging modality will have the highest yield is not known. Nevertheless because of the potential to expedite a diagnosis of LCH in a patient with iCDI, whilst avoiding stalk biopsy in those with iTPS, it is worth consideration. Early data suggest that both WB-MRI (63) and FDG PET-CT $(64,65)$ may be more sensitive and accurate in detecting LCH lesions compared to conventional imaging, but their availability and the higher radiation dose for PET-CT limit their use. In the absence of data showing the superiority of one technique over another, the choice of whole-body imaging depends on local availability and patient circumstances (e.g. age).

Although there is inadequate evidence regarding the safety, indications for, and diagnostic yield of TPS biopsy in CYP, TPS that are very large $(\geq 6.5-7 \cdot 0 \mathrm{~mm})$ or associated with pituitary or visual deficits, are more likely to herald neoplasms $(4,12-15)$. Hence, these criteria are considered in the MDT decision-making process regarding a biopsy of high diagnostic yield and low morbidity; a transcranial biopsy was diagnostic without morbidity in 6/7 children (64) and a transsphenoidal biopsy in 7/7 children (though one required a repeat procedure) (66).

For patient safety and the best risk-benefit outcome, there was consensus that surgery should only be undertaken by a skilled pituitary surgeon. Pituitary surgery may cause peri-operative endocrine morbidity (triphasic response/salt wasting), including acute dehydration implicated in cerebrovascular accidents, especially in young children. Pre- and peri-operative on site paediatric endocrine support is essential, hence the need for specialist multiprofessional centre care.

\section{Panel 4: Treatment}

- When an underlying aetiology is identified, the management should be dictated by this. Do not offer empiric disease-specific treatment without a confirmed aetiological diagnosis. (Delphi 93\%)

Treatment of the potential underlying aetiologies differ markedly and the side effects of inappropriate therapy can be harmful and may potentially mask an alternative diagnosis. CNS GCT require radiotherapy with/without 
chemotherapy (67), whilst LCH requires prednisolone and chemotherapy (34). CDI induced by LCH is usually permanent and the possibility that timely systemic LCH therapy may avoid a later risk of anterior pituitary dysfunction or neurodegeneration is not yet proven (68). Hence, in the absence of evidence of benefit, and to avoid harm, the GDG felt strongly that empiric LCH therapy should not be offered without a prior histological diagnosis.

\section{Panel 5: Surveillance and transition}

Surveillance for all $\underline{C Y P}$ presenting with $\underline{\text { iTPS }}$ and/or $\underline{\text { iCDI }}$

- Offer regular surveillance including history, examination, endocrine +/- visual assessment and pituitary MRI to all CYP with iTPS and/or iCDI. (II, Delphi 100\%)

- In the absence of new symptoms or signs and if the MRI appearances are stable, consider the following frequency of surveillance: 3-monthly intervals for 6 months, 6-monthly intervals until 2-3 years and annually thereafter. (I, Delphi 100\%)

- If surveillance reveals progressive endocrinopathies and/or evolving visual disturbance and/or progressively enlarging TPS, consider repeating first and second line investigations (with or without biopsy). (II)

Reduced surveillance for CYP with stable isolated iTPS

- Consider reducing the MRI surveillance frequency and/or discharging patients if, after 5 years of stable imaging, growth and puberty are complete in CYP who presented with isolated iTPS and have no anterior or posterior pituitary dysfunction and the TPS has either normalised or stabilised at under 4.5-5. Omm in maximal diameter. (I, Delphi 100\%)

$\underline{\text { Reduced surveillance for }} \underline{\text { CYP }}$ with stable isolated $\underline{C D I}$

Familial CDI

- In CYP with isolated CDI and a documented mutation responsible for familial CDI, MRI surveillance should be discontinued. (II)

Isolated iCDI

- Consider discontinuing the MRI surveillance after three years, in CYP with isolated iCDI and no evidence of either TPS, anterior pituitary dysfunction or visual deterioration. (II)

- Offer continued regular endocrine and clinical surveillance screening for a late presenting GCT or LCH to all patients with isolated iCDI. (II)

- All CYP whose growth and puberty are complete but continue to have an endocrinopathy, iTPS $\geq 4 \cdot 5$ $5.0 \mathrm{~mm}$ or progressively enlarging iTPS, should be transferred for on-going age-appropriate, continued screening for late presenting GCT or LCH, to a specialist adult endocrine centre with experience in managing pituitary tumours. (II)

Where an aetiology is identified, this is usually within three years of surveillance $(7,17)$, though occasionally occult LCH and GCT have been detected up to 10 (7) and 20 years (69) after presentation, respectively. Significantly enlarged iTPS with/without iCDI are more concerning than marginal stalk thickening or persistently isolated iTPS $(7,9,10,17)$, but additional anterior pituitary deficits or visual disturbances increase the likelihood of neoplasia $(8,10,17,49)$.

The optimal interval for surveillance imaging is unknown, but typically, initial neuro-oncology surveillance is 36 monthly. In one study, the diagnosis of germinoma was made within 2.5 years of a systematic 6 -monthly follow-up over 3 years (32). Three-monthly scans for the first six months would ensure detection of rapidlyevolving disease and should be considered particularly in cases with a higher risk of heralding occult oncological conditions (TPS $\geq 4 \cdot 5 / 5 \cdot 0 \mathrm{~mm}$ or TPS + CDI or TPS with evolving anterior pituitary deficiencies and/or pituitary enlargement and/or visual impairment or TPS with other concomitant brain MRI abnormalities suggesting an underlying oncological disease). Subsequently, provided MRI appearances are stable or improve, we suggest 6-monthly MRI until 2-3 years and annually thereafter.

In asymptomatic patients with marginal $(<4 \cdot 5-5 \cdot 0 \mathrm{~mm})$ isolated iTPS that remains stable after five years of imaging, thickening may be incidental or a physiological variant. Discharge may be considered once growth and 
puberty are complete. However, by contrast, CYP with isolated iCDI have a slightly increased risk of neoplasia $(10,17)$ for which continued clinical vigilance was felt justified, though surveillance neuroimaging may be discontinued after 3 years (32).

To detect occult late presenting GCT or LCH and provide ongoing endocrine care, CYP with iTPS greater than $4 \cdot 5-5 \cdot 0 \mathrm{~mm}$, iTPS that is enlarging, or those with iCDI or other endocrinopathies, should be transferred to adult endocrine care once they are 16-18 years of age and growth and puberty are complete. The centre should have experience in managing pituitary tumours and a pituitary MDT for case review. The transition process should ideally start with consistent monitoring and facilitated meeting(s) between paediatric and adult service teams from that age of 13 years, prior to effected transfer to adult services (NICE transition guidance) (70). 


\section{Discussion}

These guidelines were jointly commissioned by the paediatric societies in endocrinology (BSPED) and oncology (CCLG), (in press in full on www.cclg.org) and endorsed by the RCPCH. They were deemed vital to clarify service provision for rare paediatric pituitary disease in the UK, not least because of growing concerns to differentiate occult malignancy from normal or congenitally abnormal midline variants (22) against an increasing background of referrals to tertiary endocrine centres from various (and sometimes age-inappropriate) disciplines. Our review highlighted for the first time, the difference in underlying aetiologies between CYP and adults - supporting a role for pituitary specific MDT's in CYP as in adults. The GDG and Delphi panels were agreed that, to avoid harm, service configuration should be effected to manage patients in specialist paediatric endocrine centres associated with age- appropriate pituitary and neurooncological services, ideally associated with a centrally commissioned outcome registry and virtual MDT for complex cases (11).

Given the lack of age dependent radiological norms and high-quality evidence, the GDG's first dilemma was to define a TPS, allowing for differing shapes, techniques and measurement error. After 2 consensus rounds and reconsideration of cut-off specificity, the GDG eventually defined a TPS in CYP as $4 \mathrm{~mm}$ or more at the optic chiasm, or $3 \mathrm{~mm}$ or more at the pituitary insertion (Figs. 3 and 4). However, in CYP, unlike in adults, even smaller stalk cut-offs ( $3 \mathrm{~mm}$ and $2 \mathrm{~mm}$ respectively) may warrant investigation and surveillance if associated with pituitary or visual dysfunction. While normative data still require collection in CYP, size criteria alone cannot discriminate between physiological and pathological variants.

To decide mandatory baseline investigations and to inform the subsequent decision-making tree the GDG devised (Fig. 2), it first reviewed prevalence and time to detection of underlying aetiologies in the 11 published paediatric series (684 cases) of iTPS or iCDI (Table 1) with an average of a 6-year follow-up. The recommended panel of minimally invasive tests include blood tests $(\beta-\mathrm{hCG}$ and $\alpha \mathrm{FP})$ and imaging (CXR, abdominal US and skeletal survey) which are simple and safe respective screens for GCT and LCH - the two most common and worrying occult causes of iTPS or ICDI in CYP. Dynamic anterior and posterior pituitary function testing and optometry - especially if encroaching on the chiasm - are important to diagnose occult CDI, GH and ACTH deficiency and visual deficits, whose presence increases concern for malignancy and alters decision-making tree (Fig. 2), especially in the youngest children who otherwise present late. By contrast, we were able to recommend against routine screening for TB, neurosarcoidoisis, and hypophysitis (as in adults) unless there are specific clinical concerns and target genetic testing for familial CDI in early onset isolated CDI with family history.

We also advised against 'blind' treatment and we deferred CSF sampling and whole body imaging to $2^{\text {nd }}$ line investigations as suspicion increases of occult neoplasia (Fig. 2), recognising that in this cohort there is uncertainty about the sensitivity of CSF tumour markers to detect GCT, and about the ability of whole body imaging to detect $\mathrm{LCH}$ at other sites more amenable to biopsy. Given that for some young children these procedures require general anaesthesia and that some of the whole-body imaging modalities involve ionizing radiation, the value of this approach requires auditing.

The indications for and timing of endoscopic pituitary biopsy in CYP is controversial. After reviewing two childhood series, the GDG perceived that the risk-benefit of transsphenoidal or transcranial biopsy could be optimised if undertaken in a specialist pituitary neurosurgical unit with on-site endocrine peri-operative support, the decision to proceed being taken on an individual basis with a specialist multidisciplinary pituitary and neurooncological MDT. Considering a biopsy only in markedly thickened $(6.5 \mathrm{~mm}$ or more) or enlarging stalks with additional visual or endocrine deficits which continue to pose a worrying diagnostic dilemma after repeated tests, is more likely to give a diagnostic yield without additional harm.

Finally, the GDG determined which cases of iTPS and/or iCDI required ongoing surveillance, which required transition to adult endocrine services, and which could be safely discharged. The decision-making flow chart aimed to facilitate the lead paediatric endocrinologist's surveillance (Fig. 2) and the detection of occult neoplasia in specific high-risk subgroups, alongside monitoring of growth, development and vision. The initial 3-monthly surveillance needed in the high-risk cases may be decreased gradually over time, depending on clinical stability and the subgroup risk. Cases of isolated iTPS under $4 \cdot 5-5 \cdot 0 \mathrm{~mm}$, could be discharged after growth and puberty was complete and 5 years of stable surveillance. However, we recommended continued 
clinical surveillance and adult transition in cases of stable isolated iCDI, despite discontinuation of MRI surveillance after 5 years. Any iTPS and iCDI combination with additional pituitary dysfunction not thought to be congenital, and non-familial cases of isolated iCDI, potentially harbour occult oncological disease and require adult transition in accordance with NICE guidelines for transition, for longer term surveillance.

It is hoped these first consensus guidelines will aid service configuration, reduce morbidity and late diagnosis of occult oncological disease, and provide a best practice framework in this complex, niche area of childhood pituitary disease, for endocrine, radiology, oncology and neurosurgery professionals. A nationally commissioned virtual paediatric pituitary MDT and registry to audit outcomes would further improve care and inform future updates. Research to clarify normative stalk size criteria in growing CYP and to study the accuracy of novel disease biomarkers and imaging techniques in making a diagnosis without need for a pituitary biopsy should be encouraged. 


\section{Acknowledgments}

The GDG would like to thank all stakeholders, the Project Board, Delphi panellists and our external peer reviewers for their input into this guideline. We also would like to thank the librarians of University College London and University Hospitals of Leicester NHS Trust for their help in the literature search process. We are particularly grateful to the Quality Improvement Committee Clinical Leads for Evidence Base Medicine and Appraisals at the $\mathrm{RCPCH}$, for their advice and appraisal during the guideline development process, and for the final endorsement of the guideline, and to Mr Ashley Gamble, CEO of the CCLG, for vital administrative and moral support throughout the guideline development, finally publishing it on the CCLG website (www.cclg.org). Lastly, we thank Dr Lorenzo Ugga for his depiction of normal and thickened stalks.

"Dr Johannes Vissier was an inspiring neuroncology colleague, a thoughtful mentor and kind friend to his peers and junior colleagues, as well as a driving force behind improved care for patients with LCH and germ cell tumours. It was this latter interest which brought him onto our collaborative guidelines for paediatric endocrine tumours' initiative back in 2013, and through which we, as his co-authors, came to know and admire him, eventually leading this specific guideline group to its long conclusion. That he should be felled by a malignancy himself just at its publication and at the peak of his career development - leaving a wife, young children and a thriving neuroncology service at Cambridge where he had recently been appointed - seems a cruel twist of fate he didn't deserve. We remember him with fondness and dedicate this guideline to his memory".

\section{Role of the funding source}

The guideline development was sponsored by unrestricted grants from Sandoz Pharmaceuticals, the professional societies CCLG, BSPED and The Society of British Neurological Surgeons, and the patient support groups 'Association of Multiple Endocrine Neoplastic Disorders' (AMEND), 'Success Charity - Life After Cure' and 'The Pituitary Foundation'. All except Sandoz were stakeholders. The latter had no role in guideline methodology or the final guideline recommendations.

\section{Author contributions}

HAS chaired the project board, obtained the funding and co-ordinated the GDG.

JV with MC led the GDG.

MC, JV, HAS were responsible for the study concept and design and drafted the manuscript.

$\mathrm{CB}$ and $\mathrm{KV}$ undertook the literature search.

All authors took part in the grading process, interpreted the data, revised the manuscript critically for important intellectual content, approved the final version to be published, and agreed to be accountable for all aspects of the work.

\section{Declaration of interests}

All GDG participants completed a conflicts of interest policy form adapted from NICE (71). These were reviewed and any potential conflicts of interests are listed below.

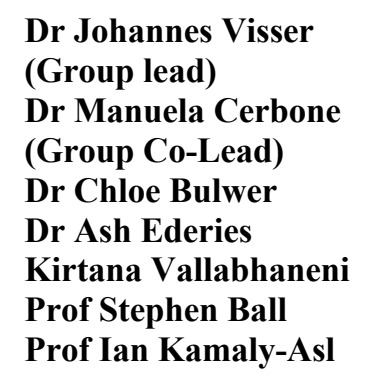

Prof Ashley Grossman Dr Helena Gleeson
None

None

None

None

None

None

Member of the NHS England Paediatric Neurosciences Clinical Reference Group 2013 onwards Member of Council of the Society of British Neurological Surgeons 2018 onwards

Chairman of the British Paediatric Neurosurgical Group 2016 to 2018

None

None 


Prof Márta Korbonits
Dr Vasanta Nanduri
Dr Vaitza Tziaferi
Prof Tom Jacques

Dr Helen Spoudeas
None

None

None

I have no direct conflict of interest but the followed are declared for transparency.

I am a director and shareholder in two private limited companies, Repath Ltd and Neuropath Ltd and I am company secretary at Repath Ltd. My wife is a director and shareholder at Neuropath Ltd. These companies handle my non-NHS work.

I am an editor in chief of the journal, Neuropathology and Applied Neurobiology.

I am the lead for the childhood solid tumour domain Genomes Clinical Interpretation Partnership

(GeCIP) for Genomes England and the 100,000 genomes projects

I have received a personal fee from Bayer for speaking on a course

Chair of the Project Board for all 8 paediatric endocrine tumours in simultaneous development Project Board Lead for Pituitary Adenomas, Craniopharyngiomas and idiopathic thickening of pituitary stalk and /or idiopathic central diabetes Insipidus

Responsible for raising grants to fund wider endeavour Initiator and founder of group with wider involvement across all guidelines Founder of 'Success Charity - Life After Cure' www.successcharity.org.uk

\section{Disclaimer}

Healthcare providers need to use clinical judgment, knowledge and expertise when deciding whether it is appropriate to apply guidelines. The recommendations cited here are a guide and may not be appropriate for use in all situations. The decision to adopt any of the recommendations cited here is the responsibility of the treating clinician and must be made in the light of individual patient circumstances, the wishes of the patient, clinical expertise and resources.

Target users of this guideline: Health professionals from a variety of paediatric and adult disciplines (including endocrinology, oncology, neurosurgery, radiology, histopathology, and genetics) involved in the diagnosis and longer term management of children and young people presenting with seemingly idiopathic pituitary stalk thickening or central diabetes insipidus within the UK. 


\section{Legends}

Fig. 1 Literature search strategy

PICO: Population, Intervention, Comparison, Outcome; iTPS: idiopathic Thickened Pituitary Stalk; iCDI: idiopathic Central Diabetes Insipidus; CYP: Children and Young People

$\S$ Full search strategy:

Text words: Thickened pituitary stalk, Pituitary stalk thickening, Pituitary stalk lesion, Central diabetes insipidus, Neurogenic diabetes insipidus, Idiopathic diabetes insipidus

Synonyms/truncation of text words: Hypophysial, Pituitary, Stalk\$, Thick\$, Diabetes Insipidus (exp), Idiopathic (exp - Embase only), Central, Neurogenic

$\sim$ Inclusion criteria: English language studies reporting on the epidemiology, clinical presentation, diagnosis, investigation, treatment or follow-up of iTPS and/or iCDI in CYP

* Exclusion criteria: pre-1990 publication, non-English or animal studies, syndromic TPS/CDI, or due to trauma/hypoxia/prematurity or pregnancy/postpartum

Fig. 2 Management flow-chart for investigation and surveillance of CYP with iTPS and/or iCDI

ESR: erythrocyte Sedimentation Rate, $\beta \mathrm{HCG}$ : beta-human chorionic gonadotrophin; $\alpha \mathrm{FP}$ : alpha-fetoprotein; GCT: germ cell tumour; HPA: hypothalamo-pituitary axis; LCH: Langerhans cell histiocytosis; XR: X-Ray; US: ultrasound; MRI: magnetic resonance images; VI: Visual impairment; +ve: FH: positive family history; CSF: cerebrospinal fluid; WB: whole body, PET: positron emission tomography, TB: tuberculosis; CYP: children and young people; SOD: septo-optic dysplasia; m: months; y: years

Fig. 3 Measurement levels of normal pituitary stalk on sagittal (A) and coronal (B) views

Stalk measurements at upper level of optic chiasm (OC) and lower level of pituitary insertion (PI) shown in bold red. A further middle measurement (dotted line) may also be taken.

Coronal images (B) should be acquired in a plane parallel to the cranio-caudal direction of the pituitary stalk as shown by the green line on sagittal views (A)

IR: Infundibular Recess, OC: Optic Chiasm, PI: Pituitary insertion, PG: Pituitary gland

Fig. 4 Different configurations of pituitary stalk thickening

Panel 1 (sagittal plane). A: Upper, B: Uniform, C: Middle, D Lower

Panel 2 (coronal plane). A: Upper, B: Uniform, C: Middle, D Lower

A: Most usual in CYP (10) 


\section{References}

1. Juul KV, Schroeder M, Rittig S, Norgaard JP. National Surveillance of Central Diabetes Insipidus (CDI) in Denmark: results from 5 years registration of 9309 prescriptions of desmopressin to 1285 CDI patients. The Journal of clinical endocrinology and metabolism. 2014;99(6):2181-7.

2. Turcu AF, Erickson BJ, Lin E, Guadalix S, Schwartz K, Scheithauer BW, et al. Pituitary stalk lesions: the Mayo Clinic experience. The Journal of clinical endocrinology and metabolism. 2013;98(5):1812-8.

3. http://www.agreetrust.org/agree-ii/.

4. $\quad$ http://www.usc.edu/hsc/ebnet/ebframe/PICO.html.

5. http://clinicalevidence.bmj.com/x/set/static/ebm/learn/665072.html.

6. http://bmjopen.bmj.com/content/6/5/e011780.full.

7. Di Iorgi N, Morana G, Maghnie M. Pituitary stalk thickening on MRI: when is the best time to re-scan and how long should we continue re-scanning for? Clinical endocrinology. 2015;83(4):449-55.

8. Robison NJ, Prabhu SP, Sun P, Chi SN, Kieran MW, Manley PE, et al. Predictors of neoplastic disease in children with isolated pituitary stalk thickening. Pediatric blood \& cancer. 2013;60(10):1630-5.

9. Leger J, Velasquez A, Garel C, Hassan M, Czernichow P. Thickened pituitary stalk on magnetic resonance imaging in children with central diabetes insipidus. The Journal of clinical endocrinology and metabolism. 1999;84(6):1954-60.

10. Cerbone M EA, Losa L, Moreno C, Spoudeas HA. Neuroimaging and eventual etiology of children with Idiopathic Thickened Pituitary Stalk (ITPS) and/or Idiopathic Central Diabetes Insipidus (ICDI): A single center experience on 53 children presenting over 30 years. Neuro-oncology. 2016;18.

11. Freund K MN, Dastamani A, Dorward N, Aquilina K, Chang YC, Mankad K, Pettorini B, Blair JJ, Kamali I, Clayton PE, Hargrave D, Korbonits M, Spoudeas HA. A 7-year update report of a national, interdisciplinary endeavour to improve outcomes for children and young people under 19 years of age with hypothalamic pituitary axis tumours (HPAT) using multi-site video conferencing. Hormone research in paediatrics. 2018;82.

12. Peyster RG, Hoover ED, Adler LP. CT of the normal pituitary stalk. AJNR American journal of neuroradiology. 1984;5(1):45-7.

13. Tien RD, Newton TH, McDermott MW, Dillon WP, Kucharczyk J. Thickened pituitary stalk on MR images in patients with diabetes insipidus and Langerhans cell histiocytosis. AJNR American journal of neuroradiology. 1990;11(4):703-8.

14. Simmons GE, Suchnicki JE, Rak KM, Damiano TR. MR imaging of the pituitary stalk: size, shape, and enhancement pattern. AJR American journal of roentgenology. 1992;159(2):375-7.

15. Satogami N, Miki Y, Koyama T, Kataoka M, Togashi K. Normal pituitary stalk: high-resolution MR imaging at 3T. AJNR American journal of neuroradiology. 2010;31(2):355-9.

16. Godano E, Morana G, Di Iorgi N, Pistorio A, Allegri AEM, Napoli F, et al. Role of MRI T2-DRIVE in the assessment of pituitary stalk abnormalities without gadolinium in pituitary diseases. European journal of endocrinology / European Federation of Endocrine Societies. 2018;178(6):613-22.

17. Werny D, Elfers C, Perez FA, Pihoker C, Roth CL. Pediatric Central Diabetes Insipidus: Brain Malformations Are Common and Few Patients Have Idiopathic Disease. The Journal of clinical endocrinology and metabolism. 2015;100(8):3074-80.

18. Bianco D, Napoli F, Morana G, Pistorio A, Allegri AEM, Fava D, et al. Endocrine Outcomes In Central Diabetes Insipidus: the Predictive Value of Neuroimaging "Mismatch Pattern". The Journal of Clinical Endocrinology \& Metabolism. 2020;105(11):3562-74.

19. Sbardella E, Joseph RN, Jafar-Mohammadi B, Isidori AM, Cudlip S, Grossman AB. Pituitary stalk thickening: the role of an innovative MRI imaging analysis which may assist in determining clinical management. European journal of endocrinology / European Federation of Endocrine Societies. 2016;175(4):255-63.

20. Ranadive SA, Ersoy B, Favre H, Cheung CC, Rosenthal SM, Miller WL, et al. Identification, characterization and rescue of a novel vasopressin-2 receptor mutation causing nephrogenic diabetes insipidus. Clinical endocrinology. 2009;71(3):388-93.

21. Klyn V, Dekeyzer S, Van Eetvelde R, Roels P, Vergauwen O, Devolder P, et al. Presence of the posterior pituitary bright spot sign on MRI in the general population: a comparison between 1.5 and 3T MRI and between 2D-T1 spin-echo- and 3D-T1 gradient-echo sequences. Pituitary. 2018;21(4):379-83.

22. Cerbone M, Guemes M, Wade A, Improda N, Dattani M. Endocrine morbidity in midline brain defects: Differences between septo-optic dysplasia and related disorders. EClinicalMedicine. 2020;19:100224.

23. Makras P, Samara C, Antoniou M, Zetos A, Papadogias D, Nikolakopoulou Z, et al. Evolving radiological features of hypothalamo-pituitary lesions in adult patients with Langerhans cell histiocytosis (LCH). Neuroradiology. 2006;48(1):37-44. 
24. Caranci F, Leone G, Ponsiglione A, Muto M, Tortora F, Muto M, et al. Imaging findings in hypophysitis: a review. Radiol Med. 2020;125(3):319-28.

25. Pal R, Rai A, Vaiphei K, Gangadhar P, Gupta P, Mukherjee KK, et al. Intracranial Germinoma Masquerading as Secondary Granulomatous Hypophysitis: A Case Report and Review of Literature. Neuroendocrinology. 2020;110(5):422-9.

26. Maghnie M. Diabetes insipidus. Hormone research. 2003;59 Suppl 1:42-54.

27. Sethi RV, Marino R, Niemierko A, Tarbell NJ, Yock TI, MacDonald SM. Delayed diagnosis in children with intracranial germ cell tumors. The Journal of pediatrics. 2013;163(5):1448-53.

28. Zhang H, Zhang P, Fan J, Qiu B, Pan J, Zhang X, et al. Determining an Optimal Cutoff of Serum betaHuman Chorionic Gonadotropin for Assisting the Diagnosis of Intracranial Germinomas. PloS one. 2016;11(1):e0147023.

29. Hu M, Guan H, Lau CC, Terashima K, Jin Z, Cui L, et al. An update on the clinical diagnostic value of $\beta$-hCG and $\alpha \mathrm{FP}$ for intracranial germ cell tumors. European journal of medical research. 2016;21:10.

30. Yoshizawa A, Yokoya S, Oyama K, Yamada S. Elevated levels of human chorionic gonadotropin-beta, a marker of active neurohypophyseal germinoma, detected by immune complex transfer enzyme immunoassay. Pituitary. 2004;7(3):165-9.

31. Fukuoka K, Yanagisawa T, Suzuki T, Shirahata M, Adachi JI, Mishima K, et al. Human chorionic gonadotropin detection in cerebrospinal fluid of patients with a germinoma and its prognostic significance: assessment by using a highly sensitive enzyme immunoassay. Journal of neurosurgery Pediatrics. 2016;18(5):573-7.

32. Di Iorgi N, Allegri AE, Napoli F, Calcagno A, Calandra E, Fratangeli N, et al. Central diabetes insipidus in children and young adults: etiological diagnosis and long-term outcome of idiopathic cases. The Journal of clinical endocrinology and metabolism. 2014;99(4):1264-72.

33. Rigaud C, Barkaoui MA, Thomas C, Bertrand Y, Lambilliotte A, Miron J, et al. Langerhans cell histiocytosis: therapeutic strategy and outcome in a 30-year nationwide cohort of 1478 patients under 18 years of age. British journal of haematology. 2016;174(6):887-98.

34. Haupt R, Minkov M, Astigarraga I, Schafer E, Nanduri V, Jubran R, et al. Langerhans cell histiocytosis $(\mathrm{LCH})$ : guidelines for diagnosis, clinical work-up, and treatment for patients till the age of 18 years. Pediatric blood \& cancer. 2013;60(2):175-84.

35. Thwaites G, Fisher M, Hemingway C, Scott G, Solomon T, Innes J. British Infection Society guidelines for the diagnosis and treatment of tuberculosis of the central nervous system in adults and children. The Journal of infection. 2009;59(3):167-87.

36. Thwaites GE, Tran TH. Tuberculous meningitis: many questions, too few answers. The Lancet Neurology. 2005;4(3):160-70.

37. Wang S, Wang L, Yao Y, Feng F, Yang H, Liang Z, et al. Primary lymphocytic hypophysitis: Clinical characteristics and treatment of 50 cases in a single centre in China over 18 years. Clinical endocrinology. 2017;87(2):177-84.

38. Honegger J, Schlaffer S, Menzel C, Droste M, Werner S, Elbelt U, et al. Diagnosis of Primary Hypophysitis in Germany. The Journal of clinical endocrinology and metabolism. 2015;100(10):3841-9. 39. Caturegli P, Newschaffer C, Olivi A, Pomper MG, Burger PC, Rose NR. Autoimmune hypophysitis. Endocrine reviews. 2005;26(5):599-614.

40. De Bellis A, Sinisi AA, Pane E, Dello Iacovo A, Bellastella G, Di Scala G, et al. Involvement of hypothalamus autoimmunity in patients with autoimmune hypopituitarism: role of antibodies to hypothalamic cells. The Journal of clinical endocrinology and metabolism. 2012;97(10):3684-90.

41. Ricciuti A, De Remigis A, Landek-Salgado MA, De Vincentiis L, Guaraldi F, Lupi I, et al. Detection of pituitary antibodies by immunofluorescence: approach and results in patients with pituitary diseases. The Journal of clinical endocrinology and metabolism. 2014;99(5):1758-66.

42. Sosa GA, Bell S, Christiansen SB, Pietrani M, Glerean M, Loto M, et al. Histologically confirmed isolated IgG4-related hypophysitis: two case reports in young women. Endocrinol Diabetes Metab Case Rep. 2014;2014:140062.

43. Li Y, Gao H, Li Z, Zhang X, Ding Y, Li F. Clinical Characteristics of 76 Patients with IgG4-Related Hypophysitis: A Systematic Literature Review. International journal of endocrinology. 2019;2019:5382640.

44. Marangoni S, Argentiero V, Tavolato B. Neurosarcoidosis. Clinical description of 7 cases with a proposal for a new diagnostic strategy. Journal of neurology. 2006;253(4):488-95.

45. Hoitsma E, Drent M, Sharma OP. A pragmatic approach to diagnosing and treating neurosarcoidosis in the 21 st century. Current opinion in pulmonary medicine. 2010;16(5):472-9.

46. Webb EA, Dattani MT. Septo-optic dysplasia. European journal of human genetics : EJHG. 2010;18(4):393-7. 
47. McCabe MJ, Dattani MT. Genetic aspects of hypothalamic and pituitary gland development. Handbook of clinical neurology. 2014;124:3-15.

48. Fang Q, George AS, Brinkmeier ML, Mortensen AH, Gergics P, Cheung LY, et al. Genetics of Combined Pituitary Hormone Deficiency: Roadmap into the Genome Era. Endocrine reviews. 2016;37(6):63675.

49. Maghnie M, Cosi G, Genovese E, Manca-Bitti ML, Cohen A, Zecca S, et al. Central diabetes insipidus in children and young adults. The New England journal of medicine. 2000;343(14):998-1007.

50. Christ-Crain M, Bichet DG, Fenske WK, Goldman MB, Rittig S, Verbalis JG, et al. Diabetes insipidus. Nature reviews Disease primers. 2019;5(1):54.

51. Allen J, Chacko J, Donahue B, Dhall G, Kretschmar C, Jakacki R, et al. Diagnostic sensitivity of serum and lumbar CSF bHCG in newly diagnosed CNS germinoma. Pediatric blood \& cancer. 2012;59(7):1180-2.

52. Kinoshita Y, Yamasaki F, Usui S, Amatya VJ, Tominaga A, Sugiyama K, et al. Solitary Langerhans cell histiocytosis located in the neurohypophysis with a positive titer HCG-beta in the cerebrospinal fluid. Child's nervous system : ChNS : official journal of the International Society for Pediatric Neurosurgery. 2016;32(5):901-4.

53. Honegger J, Mann K, Thierauf P, Zrinzo A, Fahlbusch R. Human chorionic gonadotrophin immunoactivity in cystic intracranial tumours. Clinical endocrinology. 1995;42(3):235-41.

54. Okamoto M, Yamaguchi S, Ishi Y, Motegi H, Mori T, Hashimoto T, et al. Diagnostic Capability of Cerebrospinal Fluid-Placental Alkaline Phosphatase Value in Intracranial Germ Cell Tumor. Oncology. 2020:19.

55. Murray MJ, Ajithkumar T, Harris F, Williams RM, Jalloh I, Cross J, et al. Clinical utility of circulating miR-371a-3p for the management of patients with intracranial malignant germ cell tumors. Neuro-oncology advances. 2020;2(1):vdaa048.

56. Maraka S, Janku F. BRAF alterations in primary brain tumors. Discovery medicine. 2018;26(141):51-

60.

57. García-Romero N, Carrión-Navarro J, Areal-Hidalgo P, Ortiz de Mendivil A, Asensi-Puig A, Madurga $\mathrm{R}$, et al. BRAF V600E Detection in Liquid Biopsies from Pediatric Central Nervous System Tumors. Cancers. 2019;12(1).

58. McClain KL, Picarsic J, Chakraborty R, Zinn D, Lin H, Abhyankar H, et al. CNS Langerhans cell histiocytosis: Common hematopoietic origin for $\mathrm{LCH}$-associated neurodegeneration and mass lesions. Cancer. 2018;124(12):2607-20.

59. Bridel C, Courvoisier DS, Vuilleumier N, Lalive PH. Cerebrospinal fluid angiotensin-converting enzyme for diagnosis of neurosarcoidosis. Journal of neuroimmunology. 2015;285:1-3.

60. Goo HW, Yang DH, Ra YS, Song JS, Im HJ, Seo JJ, et al. Whole-body MRI of Langerhans cell histiocytosis: comparison with radiography and bone scintigraphy. Pediatric radiology. 2006;36(10):1019-31. 61. Sher AC, Orth R, McClain K, Allen C, Hayatghaibi S, Seghers V. PET/MR in the Assessment of Pediatric Histiocytoses: A Comparison to PET/CT. Clinical nuclear medicine. 2017;42(8):582-8.

62. Phillips M, Allen C, Gerson P, McClain K. Comparison of FDG-PET scans to conventional radiography and bone scans in management of Langerhans cell histiocytosis. Pediatric blood \& cancer. 2009;52(1):97-101.

63. Kim JR, Yoon HM, Jung AY, Cho YA, Seo JJ, Lee JS. Comparison of whole-body MRI, bone scan, and radiographic skeletal survey for lesion detection and risk stratification of Langerhans Cell Histiocytosis. Scientific reports. 2019;9(1):317.

64. Albano D, Bosio G, Giubbini R, Bertagna F. Role of (18)F-FDG PET/CT in patients affected by Langerhans cell histiocytosis. Jpn J Radiol. 2017;35(10):574-83.

65. Jessop S, Crudgington D, London K, Kellie S, Howman-Giles R. FDG PET-CT in pediatric Langerhans cell histiocytosis. Pediatric blood \& cancer. 2020;67(1):e28034.

66. Mootha SL, Barkovich AJ, Grumbach MM, Edwards MS, Gitelman SE, Kaplan SL, et al. Idiopathic hypothalamic diabetes insipidus, pituitary stalk thickening, and the occult intracranial germinoma in children and adolescents. The Journal of clinical endocrinology and metabolism. 1997;82(5):1362-7.

67. Calaminus G, Kortmann R, Worch J, Nicholson JC, Alapetite C, Garre ML, et al. SIOP CNS GCT 96: final report of outcome of a prospective, multinational nonrandomized trial for children and adults with intracranial germinoma, comparing craniospinal irradiation alone with chemotherapy followed by focal primary site irradiation for patients with localized disease. Neuro-oncology. 2013;15(6):788-96.

68. Abla O, Weitzman S, Minkov M, McClain KL, Visser J, Filipovich A, et al. Diabetes insipidus in Langerhans cell histiocytosis: When is treatment indicated? Pediatric blood \& cancer. 2009;52(5):555-6. 69. Charmandari E, Brook CG. 20 years of experience in idiopathic central diabetes insipidus. Lancet. 1999;353(9171):2212-3.

70. https://research.ncl.ac.uk/transition/. 
71. https://www.nice.org.uk/about/who-we-are/policies-and-procedures. 


\section{Recommendations and explanations}

\section{Panel 1: Service Provision}

- Offer age-appropriate care, provided by an endocrinologist in a specialist centre with expertise in managing pituitary tumours, to all CYP with iTPS and/or iCDI. (I/II, Delphi 100\%)

- The endocrinologist providing care for those with iTPS and/or iCDI should liaise closely with the specialist Multidisciplinary Team (MDT) for pituitary tumours with mandated specialists from paediatric and adult endocrinology, pituitary surgery, neuroradiology, neuropathology and neurooncology. (I/II, Delphi 100\%)

- Given the rarity of pituitary tumours in CYP, a national clinical database and facilitated centralised review of images, histology and decision-making process should be developed. (Delphi 90\%)

- A centralised, national, pituitary MDT may require commissioning to facilitate review of complex cases. (Delphi 90\%)

- Offer all patients the opportunity to contribute to tissue banking and relevant ethically approved national and international biology and treatment studies. (Delphi 100\%)

Evolving pituitary dysfunction over a variable timeframe is well reported in CYP with iTPS with/without iCDI (7-10). Hence, the GDG favoured early referral to a specialist pituitary centre with an age-appropriate lead endocrinologist coordinating care, ensuring anterior and posterior pituitary assessment, and timely diagnosis and treatment of occult life-threatening endocrinopathies which affect decision making. These, and baseline optometric and radiological assessments, should be discussed at the pituitary and neuro-oncology MDT to agree management.

The limited evidence base and rarity of the condition with differing aetiology in CYP from adults, persuaded the GDG, Delphi panel and peer experts, that both a centralised pituitary MDT and a national registry would inform and help standardise care. A national forum which could form the basis of a national pituitary MDT has been operational for complex cases since 2010 (11). Such an MDT could also facilitate participation in national/international biology (with tissue banking) and treatment studies.

\section{Panel 2: Initial imaging and clinical assessment}

Defining criteria for pituitary stalk thickening

- Consider that a pituitary stalk (assessed by dedicated pituitary imaging) may be pathologically thickened and require further investigation and MRI surveillance, if there is uniform or focal thickening, in the sagittal and/or coronal plane, measuring $3 \mathrm{~mm}$ or more at pituitary insertion and/or $4 m m$ or more at the optic chiasm. (I/II, Delphi 100\%)

- Consider further investigation and MRI surveillance for stalks measuring between 2 and $3 \mathrm{~mm}$ at the pituitary insertion and/or between 3 and $4 \mathrm{~mm}$ at the level of optic chiasm if there are associated clinical features that increase the risk of pathology (such as CDI, anterior pituitary dysfunction or visual deficits). (I/II)

- $\quad$ The interpretation of the stalk appearances requires neuroradiological expertise. Given the absence of age-specific norms and the inter-and intraindividual variability, size criteria alone do not always differentiate between pathological and physiological variants. (I/II)

Dedicated pituitary MRI to detect pituitary stalk thickening

- $\quad$ Offer head and dedicated pituitary MRI to all CYP with suspected TPS and/or CDI. This should include uncontrasted $2 D$ thinly sliced $(<3 \mathrm{~mm})$ with no gap T1 and T2 weighted images in sagittal and coronal planes (and ideally at least one 3D highly weighted T2 sequence) to assess the possibility of uniform and/or focal thickening of the pituitary stalk in both planes. (II, Delphi 100\%)

Additional MRI findings which increase suspicion of pathology 
- The additional absence of a pituitary bright spot on the T1 non-contrasted scan and/or a significant reduction or enlargement of the pituitary, though not diagnostic, should increase suspicion of pathology. (II, Delphi 100\%)

- Consider a disease-tailored diagnostic approach if extra-pituitary MRI findings suggest a specific underlying aetiology (e.g. skull lesions in LCH and pineal lesions in bifocal germinoma or LCH). (II) Systematic history and clinical evaluation

- If the aetiology for a confirmed TPS and/or CDI is not apparent at presentation and a systematic history and clinical evaluation assessing the commonest causes have failed to reveal a potential focus for testing, or if focussed testing has proved uninformative, a stepwise decision-making approach for investigation and surveillance should be adopted in all patients (Fig. 2). (GDG consensus)

The stalk tapers from its top level at the optic chiasm to its lower pituitary insertion; so, measurements at upper, middle and lower levels are required to capture its normal shape and size (Fig. 3) and the degree and shape of any abnormal thickening (Fig. 4). As there are no differences between the antero-posterior and transverse diameters at all levels of measurement (12-16), the thickening of the pituitary stalk can be assessed in any direction as long as the measurements are performed in the same plane and at the same level within a protocol applied in each single centre for stalk abnormalities.

In the absence of such normative data in children, the GDG derived the dimensions of a "normal" pituitary stalk from dedicated pituitary imaging in healthy late adolescents and adult volunteers using computed tomography (12), 1.5 Tesla (T) MRI $(13,14)$ and 3T MRI $(15)$; the upper normative limits ranged between $2 \mathrm{~mm}$ and $4 \cdot 5$ $\mathrm{mm}$. Satogami et al. (15) measured the stalk at both pituitary insertion and optic chiasm on T2-weighted oblique-axial fast spin-echo images in volunteers aged 21 to 43 years. This study-reported normal dimensions of $2.32 \pm 0.39 \mathrm{~mm}$ and $2 \cdot 16 \pm 0.37 \mathrm{~mm}$ for anterior-posterior and transverse diameters respectively, at pituitary insertion, and $3.25 \pm 0.43 \mathrm{~mm}$ and $3.35 \pm 0.44 \mathrm{~mm}$ respectively, at the optic chiasm. However, Godano et al. (16), in the only paediatric study of 102 children aged 7-3-12 years, documented smaller stalk norms - between 2.35 and $2.82 \mathrm{~mm}$ proximally, 1.79 and $2.45 \mathrm{~mm}$ at midpoint and 1.28 and $1.78 \mathrm{~mm}$ distally - on T1 precontrast, T1 post-contrast, and T2-DRIVE images. However, the GDG considered these data flawed for the purpose of providing normative stalk size data in children, since the 'healthy' group included patients with congenital hypopituitarism, who, though not displaying major hypothalamo-pituitary abnormalities (without TPS and with eutopic posterior pituitary), could still have pathological congenital hypoplastic or threadlike stalks. Hence this group cannot be considered representative of the healthy population and the measurements provided might err on the lower side.

Thus, to increase the positive predictive value, the consensus was to define a TPS in CYP as measuring $3 \mathrm{~mm}$ or more at pituitary insertion and/or $4 \mathrm{~mm}$ or more at the optic chiasm, like most prior paediatric pathological case series. However, in the presence of clinical signs such as pituitary and/or visual disturbance, even smaller stalk sizes - between $2 \mathrm{~mm}$ and $3 \mathrm{~mm}$ at pituitary insertion and/or between $3 \mathrm{~mm}$ and $4 \mathrm{~mm}$ at the optic chiasm - may have pathological significance.

To differentiate pathological thickening from physiological variants, interpretation requires dedicated imaging, neuroradiological expertise and multidisciplinary assessment as well as size criteria. While very large stalks $(6 \cdot 5-7 \cdot 0 \mathrm{~mm}$ or more) are known to be more predictive of neoplasms $(7-10,17)$, the predictive value of lesser degrees of thickening is less clear, whilst how this evolves over time and its correlation with pituitary or visual deficits is unknown.

Both head and dedicated pituitary MRI are essential to accurately characterise the stalk size and shape (16), avoid artefactual misinterpretation and detect any congenital malformations.

Godano et al (16) favour high-resolution heavily T2-weighted sequences (such as sagittal T2 DRIVE which takes $<3$ minutes to acquire, or CISS or FIESTA) to precisely measure the stalk in the sagittal plane and identify its abnormalities, without the addition of gadolinium contrast (16). This, and the potential role for the so called "mismatch pattern" (consisting of discrepancies between T2-DRIVE and postcontrast T1-weighted images of PS thickness) as a prognostic marker of likelihood of evolving future anterior pituitary dysfunction or pituitary stalk stability in patients with CDI (18), is promising, but requires confirmation in further studies. 
Standard MR brain sequences must accompany the dedicated pituitary sequences to provide supporting information towards a diagnosis. Given the potential to deposit in the eloquent brain structures and in other solid organs, the use of gadolinium-based contrast medium in not routinely recommended in all children presenting with idiopathic TPS/CDI. However, its use is specifically indicated to rule out secondary brain metastases in cases where the clinical course is increasingly suspicious of a high-grade tumour, such as a germinoma (i.e. additional precocious pseudo-puberty, raised tumour markers, raised intracranial pressure, concomitant localisation in the pineal gland, basal ganglia calcification, visual abnormalities).

Additional pituitary and extra-pituitary abnormalities may aid diagnosis and should be noted (e.g. skull lesions suggestive of LCH or pineal lesions suggestive of bifocal germinoma or LCH). An absent posterior pituitary bright spot and/or anterior pituitary hypoplasia increase suspicion of pathology (19), although the former finding has also been observed in nephrogenic DI (20) and in 4.1\% of patients undergoing a brain MRI for nonendocrinological reasons (21). Suprasellar midline anomalies may indicate developmental or syndromic conditions (22). Whilst its specific prevalence in CDI is unknown, pituitary hypoplasia has been described in $98 \%$ of patients with isolated hypopituitarism and $83.3 \%$ of those with associated midline brain defects and/or optic nerve hypoplasia (22). It has also been described in $24 \%$ of LCH cases (23), whilst hypophysitis (24) and germinomas $(25,26)$ can present with pituitary enlargement.

Systematic history and clinical examination, focussed on the common differential diagnoses in CYP (Table 1 and 2), should be undertaken at diagnosis and repeatedly during follow up. If the diagnosis remains occult, we recommend a stepwise decision-making and surveillance approach (Fig. 2).

\section{Panel 3: Subsequent stepwise approach}

\section{A) First line investigations}

Serum tumour markers, haematology, liver and renal function

- Offer measurement of serum $\beta-h C G$ and $\alpha F P$ to all CYP with radiologically confirmed iTPS and/or iCDI. (II, Delphi 92\%)

- Although non-specific, consider performing Erythrocyte Sedimentation Rate (ESR), Full Blood Count $(F B C)$, liver function, urea, creatinine, and electrolytes, to aid the diagnostic process. (Delphi 92\%)

Endocrinology

- Offer an early endocrine assessment of growth and pubertal status, posterior pituitary function and baseline and dynamic tests of anterior pituitary function, including GH and ACTH reserve, to all CYP with iTPS and/or iCDI. (II, Delphi 100\%)

Ophthalmology

- Offer a formal baseline assessment of visual acuity and, if child is able to co-operate, visual fields by optometry, to all CYP with iTPS and/or iCDI, especially if the TPS is proximal to, or abutting the chiasm. (II, Delphi 100\%)

\section{Imaging}

- Offer a skeletal survey, abdominal ultrasound and a chest $x$-ray (CXR) to all CYP with iTPS and/or iCDI in whom initial blood tests have failed to reveal the aetiology. (II, Delphi 87\%)

Investigations of specific conditions as clinically indicated

Infectious and inflammatory/autoimmune disease

- Consider testing for Tuberculosis (TB) or autoimmune disease, as per local practice, if indicated by history and clinical examination. (I/II, Delphi 92\%)

- Serum Angiotensin-Converting Enzyme (ACE) to screen for neurosarcoidosis in CYP with iTPS and/or iCDI is not recommended. (I/II, Delphi 100\%)

- Congenital midline brain abnormalities [also called Septo-Optic Dysplasia (SOD) spectrum]

- Offer genetic counselling and, where appropriate, molecular genetic testing for SOD spectrum, if imaging (midline anomalies, optic nerve hypoplasia), age and ophthalmology are consistent with this diagnosis. (II)

Familial CDI 
- Offer genetic counselling and genetic testing for inherited forms of CDI in CYP with isolated CDI and neither TPS nor other midline neuroimaging abnormalities suggestive of SOD, especially if there is a family history and/or early childhood presentation. (II, Delphi 100\%)

\section{B) Second line investigations}

\section{Lumbar puncture}

Indications

- Consider a diagnostic lumbar puncture if, after initial blood tests and imaging, the aetiology is not apparent and the patient meets one or more of the following criteria a) iTPS $\geq 6 \cdot 5-7 \cdot 0 \mathrm{~mm}$ or progressively enlarging over time b) iTPS associated with iCDI c) iTPS associated with evolving anterior pituitary deficiencies and/or pituitary enlargement and/or deteriorating visual function. (II, Delphi 93\%)

Markers of GCT and LCH

- When a diagnostic lumbar puncture is undertaken, offer measurement of $\beta$ - $h C G$ and $\alpha F P$ in the CSF, together with CSF cytology. (II, Delphi 100\%)

Tuberculosis and neurosarcoidosis

- Consider CSF analysis for TB only in those at risk. (II, Delphi 78\%)

- Consider CSF ACE only if neurosarcoidosis is strongly suspected. (I, Delphi 78\%)

\section{Imaging}

- Consider whole body imaging to detect distant, occult LCH lesions more amenable to biopsy, in CYP whose TPS and/or CDI remain idiopathic after initial blood tests, imaging and CSF screening, but are nevertheless concerning for neoplasia (i.e. TPS is $\geq 6 \cdot 5-7 \cdot 0 \mathrm{~mm}$, and/or is progressive, and/or is associated with CDI, changes in pituitary size, evolving endocrinopathy, or deteriorating visual function). (II, Delphi 90\%)

- Whole body imaging may consist of FluoroDeoxy Glucose Positron Emission Tomography MRI/CT $\left({ }^{18}\right.$ FDG PET MRI or ${ }^{18}$ FDG PET CT) or whole-body MRI, depending on local availability. (II, Delphi 90\%)

\section{C) Biopsy}

- In CYP who continue to pose a diagnostic dilemma after appropriate serial neuroimaging, whole body imaging and, if necessary, repeat CSF testing, consider a biopsy of the TPS if there is a very large $(\geq 6 \cdot 5-7 \cdot 0 \mathrm{~mm})$ or progressively enlarging stalk and/or evolving hypopituitarism and/or visual deterioration. (I/II, Delphi 100\%)

- A biopsy should only be undertaken if the TPS is judged by the MDT to be of sufficient size to yield a diagnostic sample and the benefits outweigh the risk of the procedure. (Delphi 100\%)

- Pituitary surgery on CYP should be undertaken by a pituitary surgeon nominated by the MDT. There should be ready access to transsphenoidal, endoscopic and base of skull techniques and readilyavailable age-appropriate endocrine support. (I, Delphi 90\%)

Although the sensitivity and specificity of plasma $\beta-\mathrm{hCG}$ and $\alpha \mathrm{FP}$ in detecting secreting germinomas is unknown, these tumour markers are standard assessments in such patients. Their detectability and predictive value increase with repeated sampling over time, often coupled with increased stalk thickening $(7,8,27)$. Cutoff values for marker positivity differ between studies $(28,29)$, highly sensitive $\beta$-hCG assays increasing the sensitivity of disease detection $(30,31)$. Baseline ESR, FBC, urea, creatinine, electrolytes and liver function tests are included as surrogate markers of disease severity (eg LCH or inflammatory disease), and of fluid and electrolyte homeostasis in occult CDI.

Robust evidence supports frequent anterior pituitary deficits in children with iTPS or iCDI $(9,17)$ and timely comprehensive endocrine assessment will aid diagnosis and replacement therapy of occult GH deficiency, potentially life-threatening ACTH insufficiency and CDI, and normalise growth and pubertal development. Patients with isolated TPS may have occult CDI, especially if there is additional cortisol or thyroid deficiency which their replacement may unmask. The constellation and evolving hierarchy of endocrine deficits may assist differentiation of "organic" and "idiopathic" TPS and of "genetic" and "non-genetic" CDI (Fig. 2). Anterior pituitary deficits are more likely in cases with larger stalks (32) and in organic TPS (vs idiopathic forms) $(9,10$, 
32), whilst genetic CDI is usually not associated with anterior pituitary deficits (17). It is well-known that secreting germ-cell tumours can present with precocious pseudopuberty.

Pathologies underlying TPS or CDI may extend to the optic pathways and cause visual dysfunction. In a retrospective review of 53 children, $10 \%$ with isolated iTPS, $22 \%$ with isolated iCDI and $8 \%$ with both iTPS and iCDI, presented with visual symptoms (10). This increased to $35 \%$ over time only in those with both iTPS and iCDI (10), suggesting that all patients, especially the latter group, need close ophthalmology monitoring. The rate of detection of extra-pituitary LCH, using different imaging modalities in CYP with iTPS or iCDI, is unknown. However, in CYP with known $\mathrm{LCH}, 76 \cdot 8 \%$ and $7 \cdot 7 \%$ have skeletal and hepatic disease respectively (33). A possible bony LCH lesion is more accessible to diagnostic biopsy than the pituitary stalk. Hepatomegaly or splenomegaly is suspicious of LCH or other multisystem disease, whilst a CXR may identify pulmonary involvement seen in $7 \cdot 6 \%$ of LCH cases $(33,34)$ and in $50 \%$ of tuberculous meningitis cases, a rare cause of iTPS or iCDI (35).

Unlike in adults, infectious and inflammatory or autoimmune diseases are rare causes of iTPS or ICDI in CYP (Table 1). Thus, screening tests for their detection are likely to be of very low yield unless there are strong clinical indicators. Tuberculin skin testing and plasma Interferon-Gamma Release Assays may provide evidence of prior TB but are insensitive and non-specific in diagnosing active tuberculous central nervous system (CNS) disease, which requires cerebrospinal fluid (CSF) mycobacterial studies (36).

In adults with autoimmune hypophysitis, $96 \%$ have TPS and $72 \%$ have CDI $(37,38)$, but hypophysitis itself is rare in CYP (39) (Table 1). A definitive diagnosis relies on histology since neuroimaging findings are nondiscriminatory and anti-pituitary and anti-hypothalamic antibodies $(40,41)$ lack sensitivity and specificity (39). IgG4-related hypophysitis - a new entity of pituitary dysfunction and TPS, usually with multiorgan involvement (42). As yet only one of 76 described cases affected the CYP population (one 16 year old) (43), hence routine measurement of IgG4 levels are not currently recommended.

Although frequently reported in adults with iTPS (2), sarcoidosis is exceptionally rare in CYP and serum ACE lacks sensitivity and specificity to detect neurosarcoidosis $(44,45)$. Hence, we do not recommend its routine use in CYP.

Midline neuroimaging abnormalities and/or optic nerve hypoplasia associated with CDI in a young child should raise suspicion of SOD spectrum $(10,46)$. Causative mutations are identified in under $20 \%$ of such cases $(47$, 48), but SOD may account for between $3 \cdot 4 \%$ and $14 \cdot 3 \%$ of patients with CDI (Table 1). Thus, genetic counselling with appropriate testing should be offered in these cases.

Familial CDI accounts for 2\% (10) to $7 \cdot 5 \%$ (17) of CDI in CYP (Table 1). Isolated and early-presenting cases, should prompt a careful family history, genetic counselling and a targeted genetic analysis for a dominant or, rarely, a recessive variant in the arginine vasopressin $(A V P)$ gene, particularly if symptoms are familial. $(49,50)$. Wolfram syndrome due to biallelic mutations in WSF1 gene can also cause familial CDI (50). PCSK1 mutations resulting in severe malabsorptive diarrhoea, growth hormone deficiency, central hypothyroidism, central hypogonadism and central hypocortisolism have also been recently associated with clinical CDI in $80 \%$ of these cases (50). In the rare X-linked form currently no known genes have been identified.

CYP with iTPS and/or iCDI whose aetiology remains occult after the above tests, and who in addition manifest significant or progressive TPS, endocrinopathies or visual compromise, may harbour neoplasia. In two case series comprising 53 children followed for some four years, only those with both iTPS and iCDI harboured neoplasia which was detected later $(8,10)$. In cases of isolated iCDI, the risk is less clear, not least because iTPS may additionally evolve. In one series, $40 \%$ of 38 cases with isolated iCDI developed neoplasia 1.42 to 21.83 years later, but $45 \%$ of these also developed iTPS during surveillance (10). Another series reported neoplasia in $0 / 12$ cases with isolated iCDI as compared with $4 / 10$ cases with both iCDI and iTPS after $0 \cdot 6$ to $15 \cdot 3$ years (17). Thus, the GDG considered iCDI with iTPS more indicative of underlying pathology requiring a lumbar puncture than iTPS alone, whilst the evidence for isolated iCDI is inconsistent. Nevertheless, since both series report evolving anterior pituitary dysfunction more commonly in those harbouring neoplastic disease, compared with those who don't ( $85 \%$ vs $39 \%$ (4) and $67 \%$ vs $39 \%$ (10)), and visual deterioration also tracks with neoplasia (10), the presence of these signs with iTPS decreases the threshold for CSF sampling.

Using CSF levels of $\beta$-hCG, $\alpha \mathrm{FP}$ and cytology to detect GCT is well established, but their sensitivity and specificity in iTPS or iCDI is unknown. Mildly elevated CSF $\beta$-hCG is detected in up to $38 \%$ of suprasellar 
germinomas while the majority of these patients have normal serum $\beta H C G$ levels (51). Significantly raised $\beta$ $\mathrm{hCG}$ or raised $\alpha \mathrm{FP}$ (in the context of TPS) indicates the presence of choriocarcinoma and yolk sac tumour respectively, or the presence of a mixed malignant germ cell tumour. The tumour marker levels at which the diagnosis of these conditions can be made is still debated (29). It is of note that mildly elevated CSF $\beta$-hCG levels have also been described in LCH and craniopharyngioma $(52,53)$.

Several authors have recently explored novel disease markers in patients with brain tumours. Okamoto et al (54) suggest Fluid-Placental Alkaline Phosphatase (FLAP) can differentiate intracranial germ cell tumours from other types of brain tumours and detect disease recurrence, whilst Murray et al (55) highlight how microRNA quantification may assist the non-invasive diagnosis, prognostication and management of such patients. BRAFV600E alterations have been identified in various types of primary brain tumours (56) and their detection in the serum, plasma and CSF of children with brain tumours is under investigation (57); for example, whether its detection in the CSF of patients with LCH might indicate increased risk for LCH-associated neurodegeneration (58). These and other novel markers are recommended for further research.

Given the rarity of CNS tuberculous disease in causing iTPS and/or iCDI in CYP, routine CSF examination for acid-fast bacilli (36) is not recommended, but should be considered in high-risk or exposed cases. Because the diagnostic yield is lower in children than in adults (15-20\% vs $80 \%)$ and critically dependent on CSF volume, repeated CSF examinations and commercial nucleic acid amplification assays may be needed to confirm the diagnosis where there is a high index of suspicion, whilst a tissue biopsy from a tuberculoma has a higher diagnostic yield (36).

Neurosarcoidosis is unlikely to be the cause of iTPS or iCDI in CYP, and given the inconsistent reports on the clinical utility of ACE measurement in the CSF $(44,59)$, this is not recommended unless neurosarcoidosis is strongly suspected.

Whole body (WB) imaging with ${ }^{18} \mathrm{FDG}$ PET MRI/CT or MRI are not routinely used to stage patients with LCH but may detect extracranial lesions not otherwise identified (34, 60-62). If abnormalities outside the TPS are detected and suspicious, these may be easier targets for diagnostic biopsy. The accuracy of these imaging modalities to identify such lesions in this context is uncertain and which whole body imaging modality will have the highest yield is not known. Nevertheless because of the potential to expedite a diagnosis of LCH in a patient with iCDI, whilst avoiding stalk biopsy in those with iTPS, it is worth consideration. Early data suggest that both WB-MRI (63) and FDG PET-CT $(64,65)$ may be more sensitive and accurate in detecting LCH lesions compared to conventional imaging, but their availability and the higher radiation dose for PET-CT limit their use. In the absence of data showing the superiority of one technique over another, the choice of whole-body imaging depends on local availability and patient circumstances (e.g. age).

Although there is inadequate evidence regarding the safety, indications for, and diagnostic yield of TPS biopsy in CYP, TPS that are very large $(\geq 6.5-7 \cdot 0 \mathrm{~mm})$ or associated with pituitary or visual deficits, are more likely to herald neoplasms $(4,12-15)$. Hence, these criteria are considered in the MDT decision-making process regarding a biopsy of high diagnostic yield and low morbidity; a transcranial biopsy was diagnostic without morbidity in 6/7 children (64) and a transsphenoidal biopsy in 7/7 children (though one required a repeat procedure) (66).

For patient safety and the best risk-benefit outcome, there was consensus that surgery should only be undertaken by a skilled pituitary surgeon. Pituitary surgery may cause peri-operative endocrine morbidity (triphasic response/salt wasting), including acute dehydration implicated in cerebrovascular accidents, especially in young children. Pre- and peri-operative on site paediatric endocrine support is essential, hence the need for specialist multiprofessional centre care.

\section{Panel 4: Treatment}

- When an underlying aetiology is identified, the management should be dictated by this. Do not offer empiric disease-specific treatment without a confirmed aetiological diagnosis. (Delphi 93\%)

Treatment of the potential underlying aetiologies differ markedly and the side effects of inappropriate therapy can be harmful and may potentially mask an alternative diagnosis. CNS GCT require radiotherapy with/without 
chemotherapy (67), whilst LCH requires prednisolone and chemotherapy (34). CDI induced by LCH is usually permanent and the possibility that timely systemic LCH therapy may avoid a later risk of anterior pituitary dysfunction or neurodegeneration is not yet proven (68). Hence, in the absence of evidence of benefit, and to avoid harm, the GDG felt strongly that empiric LCH therapy should not be offered without a prior histological diagnosis.

\section{Panel 5: Surveillance and transition}

Surveillance for all $\underline{C Y P}$ presenting with $\underline{\text { iTPS }}$ and/or $\underline{\text { iCDI }}$

- Offer regular surveillance including history, examination, endocrine +/- visual assessment and pituitary MRI to all CYP with iTPS and/or iCDI. (II, Delphi 100\%)

- In the absence of new symptoms or signs and if the MRI appearances are stable, consider the following frequency of surveillance: 3-monthly intervals for 6 months, 6-monthly intervals until 2-3 years and annually thereafter. (I, Delphi 100\%)

- If surveillance reveals progressive endocrinopathies and/or evolving visual disturbance and/or progressively enlarging TPS, consider repeating first and second line investigations (with or without biopsy). (II)

Reduced surveillance for CYP with stable isolated iTPS

- Consider reducing the MRI surveillance frequency and/or discharging patients if, after 5 years of stable imaging, growth and puberty are complete in CYP who presented with isolated iTPS and have no anterior or posterior pituitary dysfunction and the TPS has either normalised or stabilised at under 4.5-5. Omm in maximal diameter. (I, Delphi 100\%)

$\underline{\text { Reduced surveillance for }} \underline{\text { CYP }}$ with stable isolated $\underline{C D I}$

Familial CDI

- In CYP with isolated CDI and a documented mutation responsible for familial CDI, MRI surveillance should be discontinued. (II)

Isolated iCDI

- Consider discontinuing the MRI surveillance after three years, in CYP with isolated iCDI and no evidence of either TPS, anterior pituitary dysfunction or visual deterioration. (II)

- Offer continued regular endocrine and clinical surveillance screening for a late presenting GCT or LCH to all patients with isolated iCDI. (II)

- All CYP whose growth and puberty are complete but continue to have an endocrinopathy, iTPS $\geq 4 \cdot 5$ $5.0 \mathrm{~mm}$ or progressively enlarging iTPS, should be transferred for on-going age-appropriate, continued screening for late presenting GCT or LCH, to a specialist adult endocrine centre with experience in managing pituitary tumours. (II)

Where an aetiology is identified, this is usually within three years of surveillance $(7,17)$, though occasionally occult LCH and GCT have been detected up to 10 (7) and 20 years (69) after presentation, respectively. Significantly enlarged iTPS with/without iCDI are more concerning than marginal stalk thickening or persistently isolated iTPS $(7,9,10,17)$, but additional anterior pituitary deficits or visual disturbances increase the likelihood of neoplasia $(8,10,17,49)$.

The optimal interval for surveillance imaging is unknown, but typically, initial neuro-oncology surveillance is 36 monthly. In one study, the diagnosis of germinoma was made within 2.5 years of a systematic 6 -monthly follow-up over 3 years (32). Three-monthly scans for the first six months would ensure detection of rapidlyevolving disease and should be considered particularly in cases with a higher risk of heralding occult oncological conditions (TPS $\geq 4 \cdot 5 / 5 \cdot 0 \mathrm{~mm}$ or TPS + CDI or TPS with evolving anterior pituitary deficiencies and/or pituitary enlargement and/or visual impairment or TPS with other concomitant brain MRI abnormalities suggesting an underlying oncological disease). Subsequently, provided MRI appearances are stable or improve, we suggest 6-monthly MRI until 2-3 years and annually thereafter.

In asymptomatic patients with marginal $(<4 \cdot 5-5 \cdot 0 \mathrm{~mm})$ isolated iTPS that remains stable after five years of imaging, thickening may be incidental or a physiological variant. Discharge may be considered once growth and 
puberty are complete. However, by contrast, CYP with isolated iCDI have a slightly increased risk of neoplasia $(10,17)$ for which continued clinical vigilance was felt justified, though surveillance neuroimaging may be discontinued after 3 years (32).

To detect occult late presenting GCT or LCH and provide ongoing endocrine care, CYP with iTPS greater than $4 \cdot 5-5 \cdot 0 \mathrm{~mm}$, iTPS that is enlarging, or those with iCDI or other endocrinopathies, should be transferred to adult endocrine care once they are 16-18 years of age and growth and puberty are complete. The centre should have experience in managing pituitary tumours and a pituitary MDT for case review. The transition process should ideally start with consistent monitoring and facilitated meeting(s) between paediatric and adult service teams from that age of 13 years, prior to effected transfer to adult services (NICE transition guidance) (70). 


\section{Discussion}

These guidelines were jointly commissioned by the paediatric societies in endocrinology (BSPED) and oncology (CCLG), (in press in full on www.cclg.org) and endorsed by the RCPCH. They were deemed vital to clarify service provision for rare paediatric pituitary disease in the UK, not least because of growing concerns to differentiate occult malignancy from normal or congenitally abnormal midline variants (22) against an increasing background of referrals to tertiary endocrine centres from various (and sometimes age-inappropriate) disciplines. Our review highlighted for the first time, the difference in underlying aetiologies between CYP and adults - supporting a role for pituitary specific MDT's in CYP as in adults. The GDG and Delphi panels were agreed that, to avoid harm, service configuration should be effected to manage patients in specialist paediatric endocrine centres associated with age- appropriate pituitary and neurooncological services, ideally associated with a centrally commissioned outcome registry and virtual MDT for complex cases (11).

Given the lack of age dependent radiological norms and high-quality evidence, the GDG's first dilemma was to define a TPS, allowing for differing shapes, techniques and measurement error. After 2 consensus rounds and reconsideration of cut-off specificity, the GDG eventually defined a TPS in CYP as $4 \mathrm{~mm}$ or more at the optic chiasm, or $3 \mathrm{~mm}$ or more at the pituitary insertion (Figs. 3 and 4). However, in CYP, unlike in adults, even smaller stalk cut-offs ( $3 \mathrm{~mm}$ and $2 \mathrm{~mm}$ respectively) may warrant investigation and surveillance if associated with pituitary or visual dysfunction. While normative data still require collection in CYP, size criteria alone cannot discriminate between physiological and pathological variants.

To decide mandatory baseline investigations and to inform the subsequent decision-making tree the GDG devised (Fig. 2), it first reviewed prevalence and time to detection of underlying aetiologies in the 11 published paediatric series (684 cases) of iTPS or iCDI (Table 1) with an average of a 6-year follow-up. The recommended panel of minimally invasive tests include blood tests $(\beta-\mathrm{hCG}$ and $\alpha \mathrm{FP})$ and imaging (CXR, abdominal US and skeletal survey) which are simple and safe respective screens for GCT and LCH - the two most common and worrying occult causes of iTPS or ICDI in CYP. Dynamic anterior and posterior pituitary function testing and optometry - especially if encroaching on the chiasm - are important to diagnose occult CDI, GH and ACTH deficiency and visual deficits, whose presence increases concern for malignancy and alters decision-making tree (Fig. 2), especially in the youngest children who otherwise present late. By contrast, we were able to recommend against routine screening for TB, neurosarcoidoisis, and hypophysitis (as in adults) unless there are specific clinical concerns and target genetic testing for familial CDI in early onset isolated CDI with family history.

We also advised against 'blind' treatment and we deferred CSF sampling and whole body imaging to $2^{\text {nd }}$ line investigations as suspicion increases of occult neoplasia (Fig. 2), recognising that in this cohort there is uncertainty about the sensitivity of CSF tumour markers to detect GCT, and about the ability of whole body imaging to detect $\mathrm{LCH}$ at other sites more amenable to biopsy. Given that for some young children these procedures require general anaesthesia and that some of the whole-body imaging modalities involve ionizing radiation, the value of this approach requires auditing.

The indications for and timing of endoscopic pituitary biopsy in CYP is controversial. After reviewing two childhood series, the GDG perceived that the risk-benefit of transsphenoidal or transcranial biopsy could be optimised if undertaken in a specialist pituitary neurosurgical unit with on-site endocrine peri-operative support, the decision to proceed being taken on an individual basis with a specialist multidisciplinary pituitary and neurooncological MDT. Considering a biopsy only in markedly thickened $(6.5 \mathrm{~mm}$ or more) or enlarging stalks with additional visual or endocrine deficits which continue to pose a worrying diagnostic dilemma after repeated tests, is more likely to give a diagnostic yield without additional harm.

Finally, the GDG determined which cases of iTPS and/or iCDI required ongoing surveillance, which required transition to adult endocrine services, and which could be safely discharged. The decision-making flow chart aimed to facilitate the lead paediatric endocrinologist's surveillance (Fig. 2) and the detection of occult neoplasia in specific high-risk subgroups, alongside monitoring of growth, development and vision. The initial 3-monthly surveillance needed in the high-risk cases may be decreased gradually over time, depending on clinical stability and the subgroup risk. Cases of isolated iTPS under $4 \cdot 5-5 \cdot 0 \mathrm{~mm}$, could be discharged after growth and puberty was complete and 5 years of stable surveillance. However, we recommended continued 
clinical surveillance and adult transition in cases of stable isolated iCDI, despite discontinuation of MRI surveillance after 5 years. Any iTPS and iCDI combination with additional pituitary dysfunction not thought to be congenital, and non-familial cases of isolated iCDI, potentially harbour occult oncological disease and require adult transition in accordance with NICE guidelines for transition, for longer term surveillance.

It is hoped these first consensus guidelines will aid service configuration, reduce morbidity and late diagnosis of occult oncological disease, and provide a best practice framework in this complex, niche area of childhood pituitary disease, for endocrine, radiology, oncology and neurosurgery professionals. A nationally commissioned virtual paediatric pituitary MDT and registry to audit outcomes would further improve care and inform future updates. Research to clarify normative stalk size criteria in growing CYP and to study the accuracy of novel disease biomarkers and imaging techniques in making a diagnosis without need for a pituitary biopsy should be encouraged. 


\section{Acknowledgments}

The GDG would like to thank all stakeholders, the Project Board, Delphi panellists and our external peer reviewers for their input into this guideline. We also would like to thank the librarians of University College London and University Hospitals of Leicester NHS Trust for their help in the literature search process. We are particularly grateful to the Quality Improvement Committee Clinical Leads for Evidence Base Medicine and Appraisals at the $\mathrm{RCPCH}$, for their advice and appraisal during the guideline development process, and for the final endorsement of the guideline, and to Mr Ashley Gamble, CEO of the CCLG, for vital administrative and moral support throughout the guideline development, finally publishing it on the CCLG website (www.cclg.org). Lastly, we thank Dr Lorenzo Ugga for his depiction of normal and thickened stalks.

"Dr Johannes Vissier was an inspiring neuroncology colleague, a thoughtful mentor and kind friend to his peers and junior colleagues, as well as a driving force behind improved care for patients with LCH and germ cell tumours. It was this latter interest which brought him onto our collaborative guidelines for paediatric endocrine tumours' initiative back in 2013, and through which we, as his co-authors, came to know and admire him, eventually leading this specific guideline group to its long conclusion. That he should be felled by a malignancy himself just at its publication and at the peak of his career development - leaving a wife, young children and a thriving neuroncology service at Cambridge where he had recently been appointed - seems a cruel twist of fate he didn't deserve. We remember him with fondness and dedicate this guideline to his memory".

\section{Role of the funding source}

The guideline development was sponsored by unrestricted grants from Sandoz Pharmaceuticals, the professional societies CCLG, BSPED and The Society of British Neurological Surgeons, and the patient support groups 'Association of Multiple Endocrine Neoplastic Disorders' (AMEND), 'Success Charity - Life After Cure' and 'The Pituitary Foundation'. All except Sandoz were stakeholders. The latter had no role in guideline methodology or the final guideline recommendations.

\section{Author contributions}

HAS chaired the project board, obtained the funding and co-ordinated the GDG.

JV with MC led the GDG.

MC, JV, HAS were responsible for the study concept and design and drafted the manuscript.

$\mathrm{CB}$ and $\mathrm{KV}$ undertook the literature search.

All authors took part in the grading process, interpreted the data, revised the manuscript critically for important intellectual content, approved the final version to be published, and agreed to be accountable for all aspects of the work.

\section{Declaration of interests}

All GDG participants completed a conflicts of interest policy form adapted from NICE (71). These were reviewed and any potential conflicts of interests are listed below.

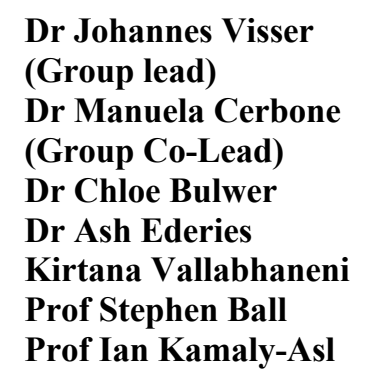

Prof Ashley Grossman Dr Helena Gleeson
None

None

None

None

None

None

Member of the NHS England Paediatric Neurosciences Clinical Reference Group 2013 onwards Member of Council of the Society of British Neurological Surgeons 2018 onwards

Chairman of the British Paediatric Neurosurgical Group 2016 to 2018

None

None 


Prof Márta Korbonits
Dr Vasanta Nanduri
Dr Vaitza Tziaferi
Prof Tom Jacques

Dr Helen Spoudeas
None

None

None

I have no direct conflict of interest but the followed are declared for transparency.

I am a director and shareholder in two private limited companies, Repath Ltd and Neuropath Ltd and I am company secretary at Repath Ltd. My wife is a director and shareholder at Neuropath Ltd. These companies handle my non-NHS work.

I am an editor in chief of the journal, Neuropathology and Applied Neurobiology.

I am the lead for the childhood solid tumour domain Genomes Clinical Interpretation Partnership

(GeCIP) for Genomes England and the 100,000 genomes projects

I have received a personal fee from Bayer for speaking on a course

Chair of the Project Board for all 8 paediatric endocrine tumours in simultaneous development Project Board Lead for Pituitary Adenomas, Craniopharyngiomas and idiopathic thickening of pituitary stalk and /or idiopathic central diabetes Insipidus

Responsible for raising grants to fund wider endeavour Initiator and founder of group with wider involvement across all guidelines Founder of 'Success Charity - Life After Cure' www.successcharity.org.uk

\section{Disclaimer}

Healthcare providers need to use clinical judgment, knowledge and expertise when deciding whether it is appropriate to apply guidelines. The recommendations cited here are a guide and may not be appropriate for use in all situations. The decision to adopt any of the recommendations cited here is the responsibility of the treating clinician and must be made in the light of individual patient circumstances, the wishes of the patient, clinical expertise and resources.

Target users of this guideline: Health professionals from a variety of paediatric and adult disciplines (including endocrinology, oncology, neurosurgery, radiology, histopathology, and genetics) involved in the diagnosis and longer term management of children and young people presenting with seemingly idiopathic pituitary stalk thickening or central diabetes insipidus within the UK. 


\section{Legends}

Fig. 1 Literature search strategy

PICO: Population, Intervention, Comparison, Outcome; iTPS: idiopathic Thickened Pituitary Stalk; iCDI: idiopathic Central Diabetes Insipidus; CYP: Children and Young People

$\S$ Full search strategy:

Text words: Thickened pituitary stalk, Pituitary stalk thickening, Pituitary stalk lesion, Central diabetes insipidus, Neurogenic diabetes insipidus, Idiopathic diabetes insipidus

Synonyms/truncation of text words: Hypophysial, Pituitary, Stalk\$, Thick\$, Diabetes Insipidus (exp), Idiopathic (exp - Embase only), Central, Neurogenic

$\sim$ Inclusion criteria: English language studies reporting on the epidemiology, clinical presentation, diagnosis, investigation, treatment or follow-up of iTPS and/or iCDI in CYP

* Exclusion criteria: pre-1990 publication, non-English or animal studies, syndromic TPS/CDI, or due to trauma/hypoxia/prematurity or pregnancy/postpartum

Fig. 2 Management flow-chart for investigation and surveillance of CYP with iTPS and/or iCDI

ESR: erythrocyte Sedimentation Rate, $\beta \mathrm{HCG}$ : beta-human chorionic gonadotrophin; $\alpha \mathrm{FP}$ : alpha-fetoprotein; GCT: germ cell tumour; HPA: hypothalamo-pituitary axis; LCH: Langerhans cell histiocytosis; XR: X-Ray; US: ultrasound; MRI: magnetic resonance images; VI: Visual impairment; +ve: FH: positive family history; CSF: cerebrospinal fluid; WB: whole body, PET: positron emission tomography, TB: tuberculosis; CYP: children and young people; SOD: septo-optic dysplasia; m: months; y: years

Fig. 3 Measurement levels of normal pituitary stalk on sagittal (A) and coronal (B) views

Stalk measurements at upper level of optic chiasm (OC) and lower level of pituitary insertion (PI) shown in bold red. A further middle measurement (dotted line) may also be taken.

Coronal images (B) should be acquired in a plane parallel to the cranio-caudal direction of the pituitary stalk as shown by the green line on sagittal views (A)

IR: Infundibular Recess, OC: Optic Chiasm, PI: Pituitary insertion, PG: Pituitary gland

Fig. 4 Different configurations of pituitary stalk thickening

Panel 1 (sagittal plane). A: Upper, B: Uniform, C: Middle, D Lower

Panel 2 (coronal plane). A: Upper, B: Uniform, C: Middle, D Lower

A: Most usual in CYP (10) 


\section{References}

1. Juul KV, Schroeder M, Rittig S, Norgaard JP. National Surveillance of Central Diabetes Insipidus (CDI) in Denmark: results from 5 years registration of 9309 prescriptions of desmopressin to 1285 CDI patients. The Journal of clinical endocrinology and metabolism. 2014;99(6):2181-7.

2. Turcu AF, Erickson BJ, Lin E, Guadalix S, Schwartz K, Scheithauer BW, et al. Pituitary stalk lesions: the Mayo Clinic experience. The Journal of clinical endocrinology and metabolism. 2013;98(5):1812-8.

3. http://www.agreetrust.org/agree-ii/.

4. $\quad$ http://www.usc.edu/hsc/ebnet/ebframe/PICO.html.

5. http://clinicalevidence.bmj.com/x/set/static/ebm/learn/665072.html.

6. http://bmjopen.bmj.com/content/6/5/e011780.full.

7. Di Iorgi N, Morana G, Maghnie M. Pituitary stalk thickening on MRI: when is the best time to re-scan and how long should we continue re-scanning for? Clinical endocrinology. 2015;83(4):449-55.

8. Robison NJ, Prabhu SP, Sun P, Chi SN, Kieran MW, Manley PE, et al. Predictors of neoplastic disease in children with isolated pituitary stalk thickening. Pediatric blood \& cancer. 2013;60(10):1630-5.

9. Leger J, Velasquez A, Garel C, Hassan M, Czernichow P. Thickened pituitary stalk on magnetic resonance imaging in children with central diabetes insipidus. The Journal of clinical endocrinology and metabolism. 1999;84(6):1954-60.

10. Cerbone M EA, Losa L, Moreno C, Spoudeas HA. Neuroimaging and eventual etiology of children with Idiopathic Thickened Pituitary Stalk (ITPS) and/or Idiopathic Central Diabetes Insipidus (ICDI): A single center experience on 53 children presenting over 30 years. Neuro-oncology. 2016;18.

11. Freund K MN, Dastamani A, Dorward N, Aquilina K, Chang YC, Mankad K, Pettorini B, Blair JJ, Kamali I, Clayton PE, Hargrave D, Korbonits M, Spoudeas HA. A 7-year update report of a national, interdisciplinary endeavour to improve outcomes for children and young people under 19 years of age with hypothalamic pituitary axis tumours (HPAT) using multi-site video conferencing. Hormone research in paediatrics. 2018;82.

12. Peyster RG, Hoover ED, Adler LP. CT of the normal pituitary stalk. AJNR American journal of neuroradiology. 1984;5(1):45-7.

13. Tien RD, Newton TH, McDermott MW, Dillon WP, Kucharczyk J. Thickened pituitary stalk on MR images in patients with diabetes insipidus and Langerhans cell histiocytosis. AJNR American journal of neuroradiology. 1990;11(4):703-8.

14. Simmons GE, Suchnicki JE, Rak KM, Damiano TR. MR imaging of the pituitary stalk: size, shape, and enhancement pattern. AJR American journal of roentgenology. 1992;159(2):375-7.

15. Satogami N, Miki Y, Koyama T, Kataoka M, Togashi K. Normal pituitary stalk: high-resolution MR imaging at 3T. AJNR American journal of neuroradiology. 2010;31(2):355-9.

16. Godano E, Morana G, Di Iorgi N, Pistorio A, Allegri AEM, Napoli F, et al. Role of MRI T2-DRIVE in the assessment of pituitary stalk abnormalities without gadolinium in pituitary diseases. European journal of endocrinology / European Federation of Endocrine Societies. 2018;178(6):613-22.

17. Werny D, Elfers C, Perez FA, Pihoker C, Roth CL. Pediatric Central Diabetes Insipidus: Brain Malformations Are Common and Few Patients Have Idiopathic Disease. The Journal of clinical endocrinology and metabolism. 2015;100(8):3074-80.

18. Bianco D, Napoli F, Morana G, Pistorio A, Allegri AEM, Fava D, et al. Endocrine Outcomes In Central Diabetes Insipidus: the Predictive Value of Neuroimaging "Mismatch Pattern". The Journal of Clinical Endocrinology \& Metabolism. 2020;105(11):3562-74.

19. Sbardella E, Joseph RN, Jafar-Mohammadi B, Isidori AM, Cudlip S, Grossman AB. Pituitary stalk thickening: the role of an innovative MRI imaging analysis which may assist in determining clinical management. European journal of endocrinology / European Federation of Endocrine Societies. 2016;175(4):255-63.

20. Ranadive SA, Ersoy B, Favre H, Cheung CC, Rosenthal SM, Miller WL, et al. Identification, characterization and rescue of a novel vasopressin-2 receptor mutation causing nephrogenic diabetes insipidus. Clinical endocrinology. 2009;71(3):388-93.

21. Klyn V, Dekeyzer S, Van Eetvelde R, Roels P, Vergauwen O, Devolder P, et al. Presence of the posterior pituitary bright spot sign on MRI in the general population: a comparison between 1.5 and 3T MRI and between 2D-T1 spin-echo- and 3D-T1 gradient-echo sequences. Pituitary. 2018;21(4):379-83.

22. Cerbone M, Guemes M, Wade A, Improda N, Dattani M. Endocrine morbidity in midline brain defects: Differences between septo-optic dysplasia and related disorders. EClinicalMedicine. 2020;19:100224.

23. Makras P, Samara C, Antoniou M, Zetos A, Papadogias D, Nikolakopoulou Z, et al. Evolving radiological features of hypothalamo-pituitary lesions in adult patients with Langerhans cell histiocytosis (LCH). Neuroradiology. 2006;48(1):37-44. 
24. Caranci F, Leone G, Ponsiglione A, Muto M, Tortora F, Muto M, et al. Imaging findings in hypophysitis: a review. Radiol Med. 2020;125(3):319-28.

25. Pal R, Rai A, Vaiphei K, Gangadhar P, Gupta P, Mukherjee KK, et al. Intracranial Germinoma Masquerading as Secondary Granulomatous Hypophysitis: A Case Report and Review of Literature. Neuroendocrinology. 2020;110(5):422-9.

26. Maghnie M. Diabetes insipidus. Hormone research. 2003;59 Suppl 1:42-54.

27. Sethi RV, Marino R, Niemierko A, Tarbell NJ, Yock TI, MacDonald SM. Delayed diagnosis in children with intracranial germ cell tumors. The Journal of pediatrics. 2013;163(5):1448-53.

28. Zhang H, Zhang P, Fan J, Qiu B, Pan J, Zhang X, et al. Determining an Optimal Cutoff of Serum betaHuman Chorionic Gonadotropin for Assisting the Diagnosis of Intracranial Germinomas. PloS one. 2016;11(1):e0147023.

29. Hu M, Guan H, Lau CC, Terashima K, Jin Z, Cui L, et al. An update on the clinical diagnostic value of $\beta$-hCG and $\alpha \mathrm{FP}$ for intracranial germ cell tumors. European journal of medical research. 2016;21:10.

30. Yoshizawa A, Yokoya S, Oyama K, Yamada S. Elevated levels of human chorionic gonadotropin-beta, a marker of active neurohypophyseal germinoma, detected by immune complex transfer enzyme immunoassay. Pituitary. 2004;7(3):165-9.

31. Fukuoka K, Yanagisawa T, Suzuki T, Shirahata M, Adachi JI, Mishima K, et al. Human chorionic gonadotropin detection in cerebrospinal fluid of patients with a germinoma and its prognostic significance: assessment by using a highly sensitive enzyme immunoassay. Journal of neurosurgery Pediatrics. 2016;18(5):573-7.

32. Di Iorgi N, Allegri AE, Napoli F, Calcagno A, Calandra E, Fratangeli N, et al. Central diabetes insipidus in children and young adults: etiological diagnosis and long-term outcome of idiopathic cases. The Journal of clinical endocrinology and metabolism. 2014;99(4):1264-72.

33. Rigaud C, Barkaoui MA, Thomas C, Bertrand Y, Lambilliotte A, Miron J, et al. Langerhans cell histiocytosis: therapeutic strategy and outcome in a 30-year nationwide cohort of 1478 patients under 18 years of age. British journal of haematology. 2016;174(6):887-98.

34. Haupt R, Minkov M, Astigarraga I, Schafer E, Nanduri V, Jubran R, et al. Langerhans cell histiocytosis $(\mathrm{LCH})$ : guidelines for diagnosis, clinical work-up, and treatment for patients till the age of 18 years. Pediatric blood \& cancer. 2013;60(2):175-84.

35. Thwaites G, Fisher M, Hemingway C, Scott G, Solomon T, Innes J. British Infection Society guidelines for the diagnosis and treatment of tuberculosis of the central nervous system in adults and children. The Journal of infection. 2009;59(3):167-87.

36. Thwaites GE, Tran TH. Tuberculous meningitis: many questions, too few answers. The Lancet Neurology. 2005;4(3):160-70.

37. Wang S, Wang L, Yao Y, Feng F, Yang H, Liang Z, et al. Primary lymphocytic hypophysitis: Clinical characteristics and treatment of 50 cases in a single centre in China over 18 years. Clinical endocrinology. 2017;87(2):177-84.

38. Honegger J, Schlaffer S, Menzel C, Droste M, Werner S, Elbelt U, et al. Diagnosis of Primary Hypophysitis in Germany. The Journal of clinical endocrinology and metabolism. 2015;100(10):3841-9. 39. Caturegli P, Newschaffer C, Olivi A, Pomper MG, Burger PC, Rose NR. Autoimmune hypophysitis. Endocrine reviews. 2005;26(5):599-614.

40. De Bellis A, Sinisi AA, Pane E, Dello Iacovo A, Bellastella G, Di Scala G, et al. Involvement of hypothalamus autoimmunity in patients with autoimmune hypopituitarism: role of antibodies to hypothalamic cells. The Journal of clinical endocrinology and metabolism. 2012;97(10):3684-90.

41. Ricciuti A, De Remigis A, Landek-Salgado MA, De Vincentiis L, Guaraldi F, Lupi I, et al. Detection of pituitary antibodies by immunofluorescence: approach and results in patients with pituitary diseases. The Journal of clinical endocrinology and metabolism. 2014;99(5):1758-66.

42. Sosa GA, Bell S, Christiansen SB, Pietrani M, Glerean M, Loto M, et al. Histologically confirmed isolated IgG4-related hypophysitis: two case reports in young women. Endocrinol Diabetes Metab Case Rep. 2014;2014:140062.

43. Li Y, Gao H, Li Z, Zhang X, Ding Y, Li F. Clinical Characteristics of 76 Patients with IgG4-Related Hypophysitis: A Systematic Literature Review. International journal of endocrinology. 2019;2019:5382640.

44. Marangoni S, Argentiero V, Tavolato B. Neurosarcoidosis. Clinical description of 7 cases with a proposal for a new diagnostic strategy. Journal of neurology. 2006;253(4):488-95.

45. Hoitsma E, Drent M, Sharma OP. A pragmatic approach to diagnosing and treating neurosarcoidosis in the 21 st century. Current opinion in pulmonary medicine. 2010;16(5):472-9.

46. Webb EA, Dattani MT. Septo-optic dysplasia. European journal of human genetics : EJHG. 2010;18(4):393-7. 
47. McCabe MJ, Dattani MT. Genetic aspects of hypothalamic and pituitary gland development. Handbook of clinical neurology. 2014;124:3-15.

48. Fang Q, George AS, Brinkmeier ML, Mortensen AH, Gergics P, Cheung LY, et al. Genetics of Combined Pituitary Hormone Deficiency: Roadmap into the Genome Era. Endocrine reviews. 2016;37(6):63675.

49. Maghnie M, Cosi G, Genovese E, Manca-Bitti ML, Cohen A, Zecca S, et al. Central diabetes insipidus in children and young adults. The New England journal of medicine. 2000;343(14):998-1007.

50. Christ-Crain M, Bichet DG, Fenske WK, Goldman MB, Rittig S, Verbalis JG, et al. Diabetes insipidus. Nature reviews Disease primers. 2019;5(1):54.

51. Allen J, Chacko J, Donahue B, Dhall G, Kretschmar C, Jakacki R, et al. Diagnostic sensitivity of serum and lumbar CSF bHCG in newly diagnosed CNS germinoma. Pediatric blood \& cancer. 2012;59(7):1180-2.

52. Kinoshita Y, Yamasaki F, Usui S, Amatya VJ, Tominaga A, Sugiyama K, et al. Solitary Langerhans cell histiocytosis located in the neurohypophysis with a positive titer HCG-beta in the cerebrospinal fluid. Child's nervous system : ChNS : official journal of the International Society for Pediatric Neurosurgery. 2016;32(5):901-4.

53. Honegger J, Mann K, Thierauf P, Zrinzo A, Fahlbusch R. Human chorionic gonadotrophin immunoactivity in cystic intracranial tumours. Clinical endocrinology. 1995;42(3):235-41.

54. Okamoto M, Yamaguchi S, Ishi Y, Motegi H, Mori T, Hashimoto T, et al. Diagnostic Capability of Cerebrospinal Fluid-Placental Alkaline Phosphatase Value in Intracranial Germ Cell Tumor. Oncology. 2020:19.

55. Murray MJ, Ajithkumar T, Harris F, Williams RM, Jalloh I, Cross J, et al. Clinical utility of circulating miR-371a-3p for the management of patients with intracranial malignant germ cell tumors. Neuro-oncology advances. 2020;2(1):vdaa048.

56. Maraka S, Janku F. BRAF alterations in primary brain tumors. Discovery medicine. 2018;26(141):51-

60.

57. García-Romero N, Carrión-Navarro J, Areal-Hidalgo P, Ortiz de Mendivil A, Asensi-Puig A, Madurga $\mathrm{R}$, et al. BRAF V600E Detection in Liquid Biopsies from Pediatric Central Nervous System Tumors. Cancers. 2019;12(1).

58. McClain KL, Picarsic J, Chakraborty R, Zinn D, Lin H, Abhyankar H, et al. CNS Langerhans cell histiocytosis: Common hematopoietic origin for $\mathrm{LCH}$-associated neurodegeneration and mass lesions. Cancer. 2018;124(12):2607-20.

59. Bridel C, Courvoisier DS, Vuilleumier N, Lalive PH. Cerebrospinal fluid angiotensin-converting enzyme for diagnosis of neurosarcoidosis. Journal of neuroimmunology. 2015;285:1-3.

60. Goo HW, Yang DH, Ra YS, Song JS, Im HJ, Seo JJ, et al. Whole-body MRI of Langerhans cell histiocytosis: comparison with radiography and bone scintigraphy. Pediatric radiology. 2006;36(10):1019-31. 61. Sher AC, Orth R, McClain K, Allen C, Hayatghaibi S, Seghers V. PET/MR in the Assessment of Pediatric Histiocytoses: A Comparison to PET/CT. Clinical nuclear medicine. 2017;42(8):582-8.

62. Phillips M, Allen C, Gerson P, McClain K. Comparison of FDG-PET scans to conventional radiography and bone scans in management of Langerhans cell histiocytosis. Pediatric blood \& cancer. 2009;52(1):97-101.

63. Kim JR, Yoon HM, Jung AY, Cho YA, Seo JJ, Lee JS. Comparison of whole-body MRI, bone scan, and radiographic skeletal survey for lesion detection and risk stratification of Langerhans Cell Histiocytosis. Scientific reports. 2019;9(1):317.

64. Albano D, Bosio G, Giubbini R, Bertagna F. Role of (18)F-FDG PET/CT in patients affected by Langerhans cell histiocytosis. Jpn J Radiol. 2017;35(10):574-83.

65. Jessop S, Crudgington D, London K, Kellie S, Howman-Giles R. FDG PET-CT in pediatric Langerhans cell histiocytosis. Pediatric blood \& cancer. 2020;67(1):e28034.

66. Mootha SL, Barkovich AJ, Grumbach MM, Edwards MS, Gitelman SE, Kaplan SL, et al. Idiopathic hypothalamic diabetes insipidus, pituitary stalk thickening, and the occult intracranial germinoma in children and adolescents. The Journal of clinical endocrinology and metabolism. 1997;82(5):1362-7.

67. Calaminus G, Kortmann R, Worch J, Nicholson JC, Alapetite C, Garre ML, et al. SIOP CNS GCT 96: final report of outcome of a prospective, multinational nonrandomized trial for children and adults with intracranial germinoma, comparing craniospinal irradiation alone with chemotherapy followed by focal primary site irradiation for patients with localized disease. Neuro-oncology. 2013;15(6):788-96.

68. Abla O, Weitzman S, Minkov M, McClain KL, Visser J, Filipovich A, et al. Diabetes insipidus in Langerhans cell histiocytosis: When is treatment indicated? Pediatric blood \& cancer. 2009;52(5):555-6. 69. Charmandari E, Brook CG. 20 years of experience in idiopathic central diabetes insipidus. Lancet. 1999;353(9171):2212-3.

70. https://research.ncl.ac.uk/transition/. 


\begin{tabular}{|c|c|c|c|c|c|c|c|c|c|c|c|c|}
\hline & $\begin{array}{l}\text { Werny } \\
\text { et al } \\
2015 \\
\\
\text { (n: 147) }\end{array}$ & $\begin{array}{l}\text { Richards } \\
\text { et al } 2011 \\
\\
(\mathrm{n}: 105)\end{array}$ & $\begin{array}{l}\text { Di Iorgi } \\
\text { et al } \\
2014 \\
\text { (n:78) }\end{array}$ & $\begin{array}{c}\text { Maghnie } \\
\text { et al } \\
2000 \\
\\
\text { (n:73) }\end{array}$ & $\begin{array}{l}\text { Cerbone } \\
\text { et al } \\
2015 \\
\\
\text { (n:53) }\end{array}$ & $\begin{array}{c}\text { Santipra } \\
\text { bhob } \\
\text { et al } \\
2005 \\
(\mathrm{n}: 50)\end{array}$ & $\begin{array}{l}\text { Liu } \\
\text { et al } \\
2013 \\
\\
\text { (n:48) }\end{array}$ & $\begin{array}{l}\text { Bajpai } \\
\text { et al } \\
2008 \\
\\
(\mathrm{n}: 46)\end{array}$ & $\begin{array}{l}\text { Catli } \\
\text { et al } \\
2012 \\
\\
(\mathrm{n}: 34)\end{array}$ & $\begin{array}{c}\text { Jarurata } \\
\text { nasirikul } \\
\text { et al } \\
2002 \\
\text { (n:29) }\end{array}$ & $\begin{array}{c}\text { Hamilton } \\
\text { et al } \\
2007 \\
\\
(\mathrm{n}: 21)\end{array}$ & $\begin{array}{c}\begin{array}{c}\text { Combined } \\
\text { Cohort }\end{array} \\
(\mathrm{n}: 684)\end{array}$ \\
\hline IDIOPATHIC n (\%) & $18(12 \cdot 2)$ & $12(11 \cdot 0)$ & $43(55 \cdot 1)$ & $41(56 \cdot 2)$ & $28(52 \cdot 8)$ & $7(14 \cdot 0)$ & $5(10 \cdot 4)$ & $19(41 \cdot 3)$ & $10(29 \cdot 4)$ & $16(55 \cdot 2)$ & $0(0 \cdot 0)$ & $199(29 \cdot 1)$ \\
\hline $\begin{array}{l}\text { NEOPLASTIC n (\%) } \\
\text { 1. Langherans cells histiocytosis } \\
\text { 2. Germ cell tumours } \\
\text { 3. } \quad \text { Craniopharingioma } \\
\text { 4. } \text { Other brain tumours }^{\mathrm{a}} \\
\text { 5. } \text { Metastatic disease }^{\mathrm{b}}\end{array}$ & $\begin{array}{l}\mathbf{7 8}(\mathbf{5 3} \cdot \mathbf{1}) \\
18(12 \cdot 2) \\
15(10 \cdot 2) \\
37(25 \cdot 2) \\
8(5 \cdot 4) \\
0(0 \cdot 0)\end{array}$ & $\begin{array}{c}\mathbf{5 6}(\mathbf{5 3} \cdot \mathbf{3}) \\
20(19 \cdot 0) \\
11(10 \cdot 0) \\
19(18 \cdot 0) \\
6(6 \cdot 0) \\
0(0 \cdot 0)\end{array}$ & $\begin{array}{l}27(34 \cdot 6) \\
12(15 \cdot 4) \\
9(11 \cdot 5) \\
6(7 \cdot 7) \\
0(0 \cdot 0) \\
0(0 \cdot 0)\end{array}$ & $\begin{array}{l}24(\mathbf{3 2} \cdot 9) \\
12(16 \cdot 4) \\
6(8 \cdot 2) \\
6(8 \cdot 2) \\
0(0 \cdot 0) \\
0(0 \cdot 0)\end{array}$ & $\begin{array}{l}22(\mathbf{4 1} \cdot \mathbf{5}) \\
11(20 \cdot 7) \\
9(17 \cdot 0) \\
1(1 \cdot 9) \\
1(1 \cdot 9) \\
0(0 \cdot 0)\end{array}$ & $\begin{array}{c}14(28 \cdot 0) \\
3(6 \cdot 0) \\
8(16 \cdot 0) \\
3(6 \cdot 0) \\
0(0 \cdot 0) \\
0(0 \cdot 0)\end{array}$ & $\begin{array}{l}38(\mathbf{7 9} \cdot \mathbf{2}) \\
12(25 \cdot 0) \\
20(41 \cdot 7) \\
1(2 \cdot 1) \\
5(10 \cdot 4) \\
0(0 \cdot 0)\end{array}$ & $\begin{array}{c}17(36 \cdot 9) \\
11(23 \cdot 9) \\
2(4 \cdot 3) \\
3(6 \cdot 5) \\
1(2 \cdot 2) \\
0(0 \cdot 0)\end{array}$ & $\begin{array}{l}\mathbf{1 7}(\mathbf{5 0 \cdot 0 )} \\
4(11 \cdot 8) \\
4(11 \cdot 8) \\
7(20 \cdot 6) \\
2(5 \cdot 9) \\
0(0 \cdot 0)\end{array}$ & $\begin{array}{l}10(\mathbf{3 4} \cdot \mathbf{5}) \\
3(10 \cdot 3) \\
4(13 \cdot 7) \\
1(3 \cdot 4) \\
2(6 \cdot 9) \\
0(0 \cdot 0)\end{array}$ & $\begin{array}{l}8(38 \cdot 1) \\
4(19 \cdot 0) \\
2(9 \cdot 5) \\
0(0 \cdot 0) \\
1(4 \cdot 8) \\
1(4 \cdot 8)\end{array}$ & $\begin{array}{c}311(\mathbf{4 5} \cdot \mathbf{5}) \\
110(16 \cdot 1) \\
90(13 \cdot 1) \\
84(12 \cdot 3) \\
26(3 \cdot 8) \\
1(0 \cdot 1)\end{array}$ \\
\hline $\begin{array}{ll}\text { CONGENITAL/GENETIC n }(\%) \\
\text { 1. } & \text { Rathke and pars intermedia cyst } \\
\text { 2. } & \text { Ectopic posterior pituitary } \\
\text { 3. } & \text { Septo-optic dysplasia } \\
\text { 4. } & \text { Holoprosencephaly } \\
\text { 5. } & \text { Other congenital abnormalities } \\
\text { 6. } & \text { Genetic CDI } \\
\end{array}$ & $\begin{array}{c}46(\mathbf{3 1} \cdot \mathbf{3}) \\
0(0 \cdot 0) \\
0(0 \cdot 0) \\
21(14 \cdot 3) \\
8(5 \cdot 4) \\
6(4 \cdot 1) \\
11(7 \cdot 5) \\
\end{array}$ & $\begin{array}{c}21(\mathbf{2 0 \cdot 0}) \\
0(0 \cdot 0) \\
0(0 \cdot 0) \\
8(8 \cdot 0) \\
11(10 \cdot 0) \\
2(2 \cdot 0) \\
0(0 \cdot 0) \\
\end{array}$ & $\begin{array}{l}6(7 \cdot 7) \\
0(0 \cdot 0) \\
0(0 \cdot 0) \\
3(3 \cdot 8) \\
0(0 \cdot 0) \\
0(0 \cdot 0) \\
3(3 \cdot 8) \\
\end{array}$ & $\begin{array}{l}\mathbf{5}(\mathbf{6} \cdot \mathbf{8}) \\
0(0 \cdot 0) \\
0(0 \cdot 0) \\
0(0 \cdot 0) \\
0(0 \cdot 0) \\
0(0 \cdot 0) \\
5(6 \cdot 8) \\
\end{array}$ & $\begin{array}{l}3(5 \cdot 7) \\
2(3 \cdot 8) \\
0(0 \cdot 0) \\
0(0 \cdot 0) \\
0(0 \cdot 0) \\
0(0 \cdot 0) \\
1(1 \cdot 9) \\
\end{array}$ & $\begin{array}{c}20(\mathbf{4 0 \cdot 0}) \\
0(0 \cdot 0) \\
0(0 \cdot 0) \\
7(14 \cdot 0) \\
2(4 \cdot 0) \\
11(22 \cdot 0) \\
0(0 \cdot 0) \\
\end{array}$ & $\begin{array}{l}3(6 \cdot 2) \\
0(0 \cdot 0) \\
1(2 \cdot 1) \\
0(0 \cdot 0) \\
2(4 \cdot 2) \\
0(0 \cdot 0) \\
0(0 \cdot 0) \\
\end{array}$ & $\begin{array}{l}8(\mathbf{1 7 \cdot 4}) \\
0(0 \cdot 0) \\
0(0 \cdot 0) \\
0(0 \cdot 0) \\
4(8 \cdot 7) \\
4(8 \cdot 7) \\
0(0 \cdot 0) \\
\end{array}$ & $\begin{array}{l}4(\mathbf{( 1 1 \cdot 8 )} \\
1(2 \cdot 9) \\
0(0 \cdot 0) \\
0(0 \cdot 0) \\
3(8 \cdot 8) \\
0(0 \cdot 0) \\
0(0 \cdot 0) \\
\end{array}$ & $\begin{array}{l}2(6 \cdot 9) \\
0(0 \cdot 0) \\
0(0 \cdot 0) \\
1(3 \cdot 4) \\
0(0 \cdot 0) \\
1(3 \cdot 4) \\
0(0 \cdot 0) \\
\end{array}$ & $\begin{array}{c}13(\mathbf{( 6 1} \cdot \mathbf{9}) \\
0(0 \cdot 0) \\
0(0 \cdot 0) \\
0(0 \cdot 0) \\
0(0 \cdot 0) \\
13(61 \cdot 9) \\
0(0 \cdot 0) \\
\end{array}$ & $\begin{array}{c}131(\mathbf{( 1 9} \cdot \mathbf{1}) \\
3(0 \cdot 4) \\
1(0 \cdot 1) \\
40(5 \cdot 8) \\
30(4 \cdot 4) \\
37(5 \cdot 4) \\
20(2 \cdot 9) \\
\end{array}$ \\
\hline INFECTIOUS ${ }^{\text {e }}$ (\%) & $\mathbf{0}(\mathbf{0} \cdot \mathbf{0})$ & $4(4 \cdot 0)$ & $\mathbf{0}(\mathbf{0} \cdot \mathbf{0})$ & $\mathbf{0}(\mathbf{0} \cdot \mathbf{0})$ & $\mathbf{0}(\mathbf{0} \cdot \mathbf{0})$ & $4(8 \cdot 0)$ & $2(4 \cdot 2)$ & $2(4 \cdot 3)$ & $\mathbf{0}(\mathbf{0} \cdot \mathbf{0})$ & $1(3 \cdot 4)$ & $\mathbf{0}(\mathbf{0} \cdot \mathbf{0})$ & $13(1.9)$ \\
\hline POST-TRAUMATIC n (\%) & $2(1 \cdot 4)$ & $0(0 \cdot 0)$ & $2(2 \cdot 6)$ & $2(2 \cdot 7)$ & $0(0 \cdot 0)$ & $0(0 \cdot 0)$ & $\mathbf{0}(\mathbf{0 \cdot 0})$ & $0(0.0)$ & $2(5 \cdot 9)$ & $0(0 \cdot 0)$ & $0(0 \cdot 0)$ & $8(1 \cdot 2)$ \\
\hline $\begin{array}{l}\text { INFLAMMATORY/AUTOIMMUNE } \\
\text { n (\%) }\end{array}$ & $\mathbf{0}(\mathbf{0} \cdot \mathbf{0})$ & $\mathbf{0}(\mathbf{0} \cdot \mathbf{0})$ & $\mathbf{0}(\mathbf{0} \cdot \mathbf{0})$ & $1(1 \cdot 4)$ & $\mathbf{0}(\mathbf{0} \cdot \mathbf{0})$ & $\mathbf{0}(\mathbf{0} \cdot \mathbf{0})$ & $\mathbf{0}(\mathbf{0} \cdot \mathbf{0})$ & $0(0 \cdot 0)$ & $1(2 \cdot 9)$ & $\mathbf{0}(\mathbf{0} \cdot \mathbf{0})$ & $\mathbf{0}(\mathbf{0} \cdot \mathbf{0})$ & $2(0 \cdot 3)$ \\
\hline OTHER $^{\mathrm{g}}$ (\%) & $3(2 \cdot 0)^{\pi}$ & $12(11 \cdot 4)^{*}$ & $\mathbf{0}(\mathbf{0} \cdot \mathbf{0})$ & $0(0 \cdot 0)$ & $\mathbf{0}(\mathbf{0} \cdot \mathbf{0})$ & $5(10 \cdot 0)^{\#}$ & $\mathbf{0}(\mathbf{0} \cdot \mathbf{0})$ & $\mathbf{0}(\mathbf{0} \cdot \mathbf{0})$ & $\mathbf{0}(\mathbf{0} \cdot \mathbf{0})$ & $\mathbf{0}(\mathbf{0} \cdot \mathbf{0})$ & $\mathbf{0}(\mathbf{0} \cdot \mathbf{0})$ & $20(2 \cdot 9)$ \\
\hline
\end{tabular}

Note: from the initial combined cohort of 741 patients from the 11 paediatric studies, 57 cases with post-operative CDI were excluded 
${ }^{a}$ Optic nerve glioma, neuroectodermal tumours, astrocytoma, lymphoma, pituitary adenoma, pinealoma, cavernous hemangioma, unknown nature

${ }^{\mathrm{b}}$ Glioblastoma multiforme

${ }^{\mathrm{c}}$ Hydrocephalus, small anterior pituitary/empty sella, encephalocele, fronto-nasal dysplasia, absent septum pellucidum, hydrancephaly, schizencephaly, meningomyelocele, arachnoid cyst

${ }^{\mathrm{d}} \mathrm{AVP}$ - autosomal dominant due to AVP gene mutation; Wolfram syndrome - autosomal recessive due to WSF1 gene mutations

e Tuberculous or Group B Streptococcal or E-coli meningitis/encephalitis/ventriculo-peritoneal shunt infection, pituitary abscess

${ }^{\mathrm{f}}$ Hypophysitis

${ }^{\mathrm{g}}$ In some studies different aetiologies were combined and not specified, hence the prevalence of individual causes could not be extrapolated for those studies

ๆ Inflammatory/infectious

* Post-traumatic, metastatic, Rathke cyst, cerebral palsy, congenital

\# Cerebral palsy of unknown origin 
Table 2. Presenting signs and symptoms associated with the main aetiologies of thickened pituitary stalk (TPS) and/or central diabetes insipidus (CDI) in children and young people $<19$ years (CYP)

\section{GENERAL}

1) Central/hypothalamic: headaches (even without increased intracranial pressure), weight loss, vomiting, anorexia, change in school performance, drowsiness, bulging fontanelle, lethargy, behaviour/mood change, seizures, fever or temperature instability

2) Pituitary deficits:

- $G H$ : short stature, growth deceleration with bone age delay, immature appearance compared to peers, infantile hypoglycaemia, fatigue, reduced muscular tone and increased adipose tissue, reduced bone mineral density

- $A C T H$ : fatigue, hypoglycaemia, hypotension, hyponatraemia, hyperkalaemia, muscle weakness, loss of appetite and weight loss, nausea, vomiting, behaviour/mood change, acute collapse (especially during intercurrent illness/procedure)

- TSH: reduced school performances, growth failure, weight gain, bone age delay, neurodevelopmental delay, constipation, neonatal prolonged jaundice, tiredness, cold intolerance, puffiness around the eyes, impaired memory, depression, hoarse voice, delayed puberty, dry skin, hair loss/thinning, prolonged reflexes

- $F S H / L H$ : absent or delayed puberty, primary or secondary amenorrhoea (females) (NB. paradoxical precocious pseudopuberty in children with HCG-secreting GCT despite LH/FSH deficiency), reduced bone mineral density

- AVP: polyuria (particularly nocturia in childhood or adolescence), polydipsia (particularly night thirst, sometimes manifest as inconsolable crying or sleep disturbance in infants), weight loss or failure to thrive (from occult dehydration), hypernatraemia, dehydration, delayed growth

3) Visual disturbances: reduced vision (visual acuity) and visual fields (occult in younger children), diplopia/squint

\section{SPECIFIC}

1) LCH:

- Skin: dermatitis (can mimic ‘cradle cap' and fungal infection), vesicles, ulcerative lesions, petechiae, nodules

- Bones (Single or Multiple Sites): pain, lumps, fracture, limp, decreased mobility

- Bone Marrow: pallor, fatigue, increased susceptibility to infections, bruising, bleeding

- Gastro-intestinal tract: diarrhoea, rectal bleeding, weight loss, failure to thrive, malabsorption

- Liver: hepatomegaly (usually occurs with splenomegaly), jaundice, ascites, coagulopathy

- Spleen: splenomegaly (usually occurs with hepatomegaly)

- Lungs: shortness of breath, cough

- Central nervous system: Neurodegeneration: dysmetria, tremor, ataxia, dysarthria, behavioural disturbances, cognitive disorders and/or psychosis; Tumorous lesions: raised intracranial pressure, seizures, site specific symptoms and signs e.g. hypothalamus involvement may present with temperature instability, abnormal eating patterns with weight gain and/or behavioural problems

- Mouth/Jaw/Gums: pain and swelling of face, loosening or loss of teeth, mouth ulcers, swollen or bleeding gums

- Ears: persistent/recurrent discharge, hearing loss

- Eyes: proptosis, vision loss (extremely rare)

- Lymph Nodes: enlargement, soft or hard, may be matted

2) Germinoma: precocious pseudopuberty (if HCG secreting)

3) Optic pathway gliomas: association with neurocutaneous and occasionally hormonal (GH excess)

4) manifestations from NF1

5) Congenital midline brain defects: variable association of optic nerve, hypothalamo-pituitary and corpus callosum congenital abnormalities

6) Familial CDI: early onset forms, family history of CDI

7) Tuberculosis: contact history

Meningitis: prolonged fever, vague central nervous system symptoms, encephalopathy, anorexia, failure to thrive, poor appetite, nausea, vomiting and abdominal pain, sleep disturbances Tuberculoma: often asymptomatic 
8) Hypophysitis: family history and association with autoimmune conditions (eg. Polyglandular autoimmune syndrome). A definitive diagnosis of this condition (extremely rare in childhood) can only be made obtaining histology from neurosurgical biopsy

9) APS-1: more frequently presenting with mucocutaneous candidiasis, hypoparathyroidism and adrenal insufficiency, it can also be rarely associated with CDI

HCG: human chorionic gonadotropin; GCT: germ cell tumour; LCH: Langerhans cell histiocytosis; NF1: neurofibromatosis type 1; APS-1: Autoimmune polyglandular syndrome type 1 
Fig. 1

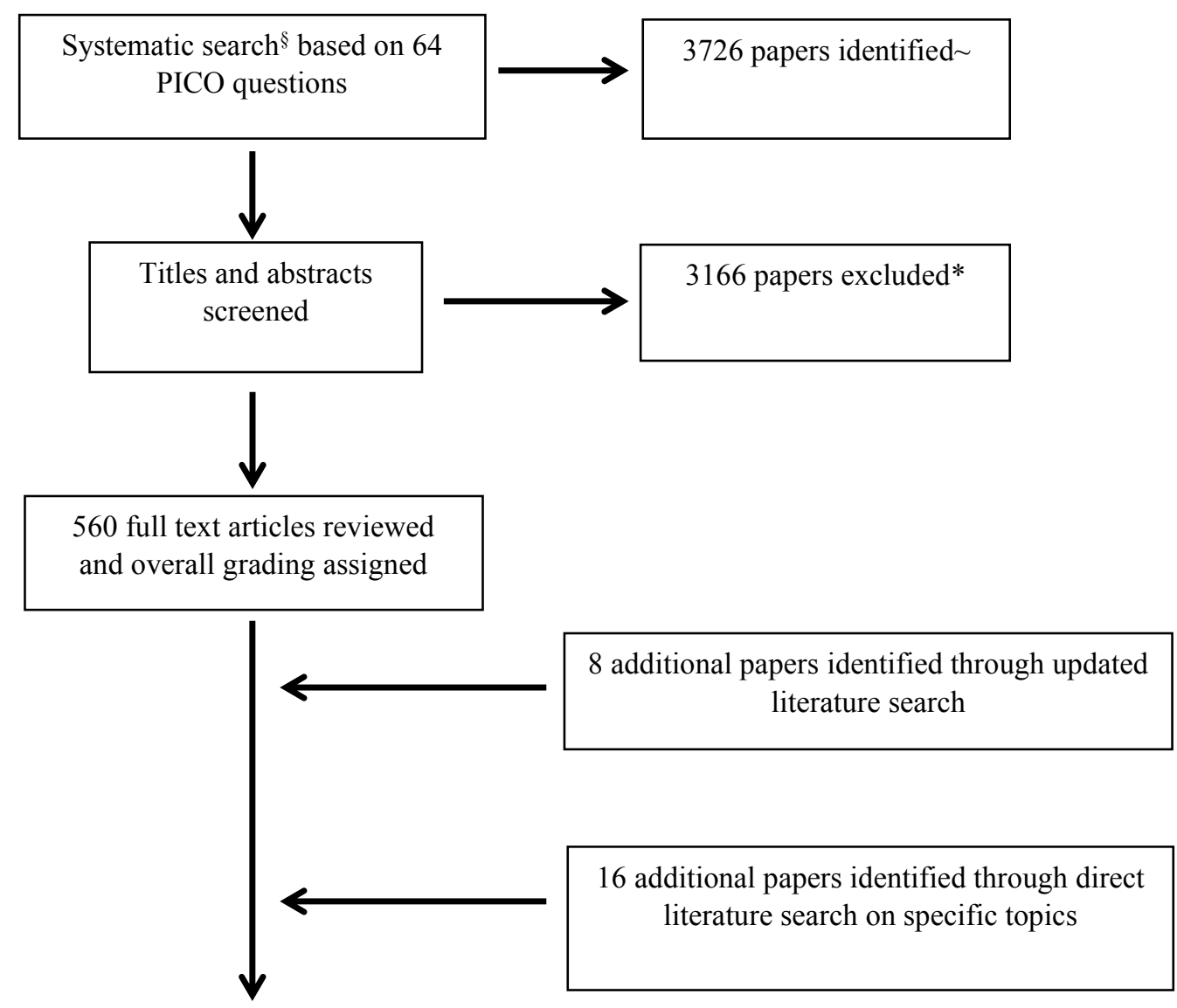

584 papers included in guideline evidence base 
Fig. 2

Idiopathic Thickened Pituitary Stalk (iTPS) and/or

Idiopathic Central Diabetes Insipidus (iCDI)

لI

History and clinical examination focussed on aetiologies

\section{II}

\#First line investigations:

Full blood count, ESR, Urea, Creatinine, Electrolytes, Liver function; $\beta H C G, \alpha$ FP for occult secreting GCT

Dynamic anterior and posterior pituitary tests for occult HPA dysfunction

- Visual acuity and visual fields - if close to chiasm

- Chest XR, Abdominal US, Skeletal Survey for occult LCH

$\sim$ Clinical (history, examination, pituitary function

+/- visual assessment) and MRI surveillance:

- 3 monthly for $6 \mathrm{~m}$, particularly in severe cases (large stalks, TPS + CDI and/or

visual/pituitary/other brain abnormalities)

- if stable, 6-monthly to 2-3y and annually thereafter MRI surveillance Consider discharge at $5 \mathrm{y}$

\section{5}
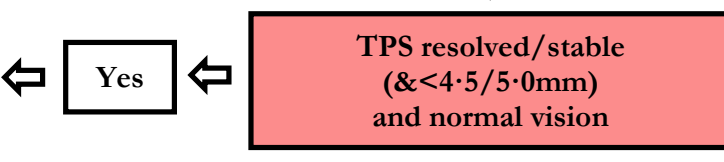

*Second line investigations:

- $\quad$ CSF cytology, $\beta$ HCG, $\alpha$ FP for occult GCT

- Consider PET/WB-MRI for occult LCH

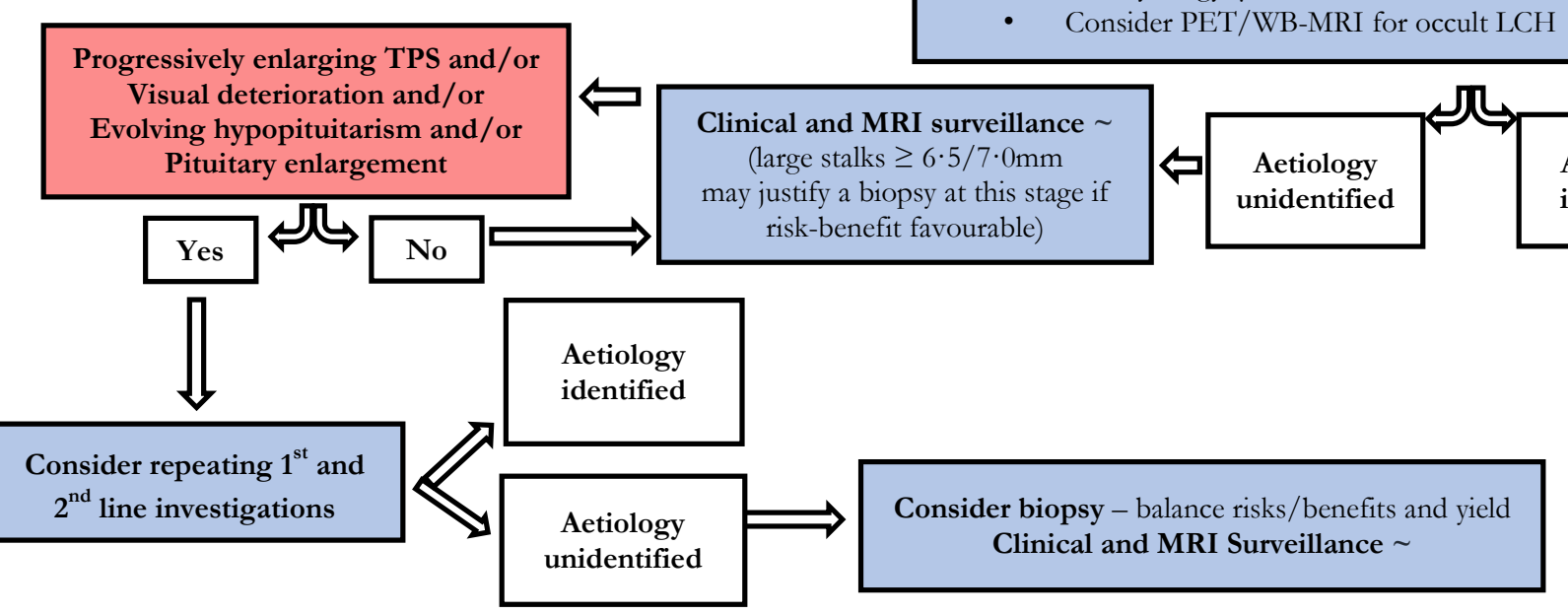

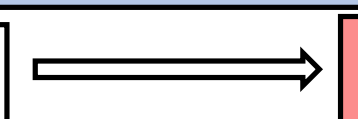

Isolated CDI

Normal stalk, vision and anterior pituitary function

$$
\sqrt{2}
$$

Clinical and MRI

surveillance

or TPS + CDI or

TPS with evolving

deficien pituitary

pituitary

enlargement

and/or visual

impairment

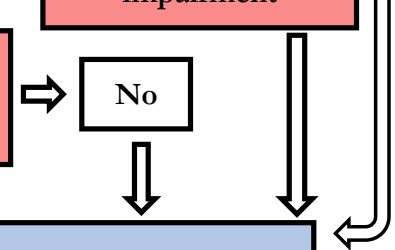

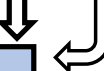

(n)

Aetiology

identified
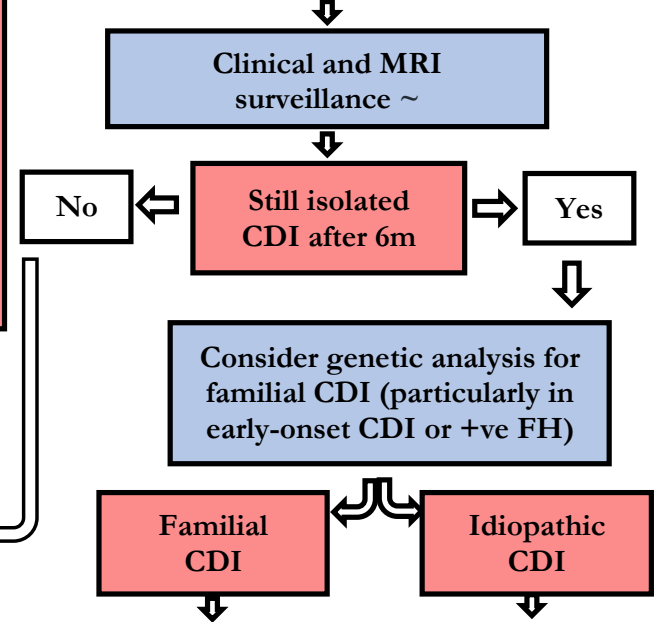

v

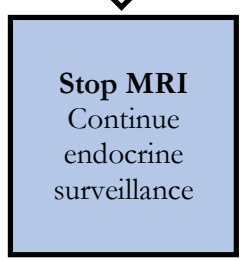

Clinical and MRI surveillance If stable and no evidence of TPS,

anterior pituitary dysfunction or visual deterioration consider discontinuing MRI surveillance after $3 y$, but continue clinical and endocrine follow-up

\section{Test for TB, neurosarcoidoisis, and hypophysitis only if specific clinical concerns Consider a diagnosis of congenital disorders (SOD spectrum) if criteria are met}

\#Investigations for specific indications in CYP:

*Second line investigations:

May be deferred in some, until after a period of clinical and MRI surveillance 


\section{Panel. Research recommendations}

- Provide normative age-appropriate references ranges for stalk measurements at three levels and explore the potential value of stalk size/shape and other imaging characteristics in distinguishing between different underlying causes

- Study the true incidence of varying aetiologies and the natural history of idiopathic TPS/CDI

- Investigate the value of novel blood and CSF markers of disease (e.g. microRNA's, cell free BRAF V600E, PLAP), and/or new imaging techniques in establishing a diagnosis without a pituitary biopsy

- Measure the impact of a systematic diagnostic and surveillance program on diagnostic yield, outcome and quality of life

- Assess the impact of early diagnosis/treatment of LCH on incidence of LCH central nervous system neurodegeneration 